\title{
Heavy quark expansion parameters from lattice NRQCD
}

\author{
S. Aoki, ${ }^{1}$ M. Fukugita, ${ }^{2}$ S. Hashimoto,${ }^{3}$ K.-I. Ishikawa, ${ }^{1,4, *}$ N. Ishizuka,,${ }^{1,4}$ Y. Iwasaki, ${ }^{1}$ K. Kanaya, ${ }^{1}$ T. Kaneko, ${ }^{3}$ \\ Y. Kuramashi, ${ }^{3}$ M. Okawa, ${ }^{5}$ N. Tsutsui, ${ }^{3}$ A. Ukawa, ${ }^{1,4}$ N. Yamada, ${ }^{3, \dagger}$ and T. Yoshie ${ }^{1,4}$ \\ (JLQCD Collaboration) \\ ${ }^{1}$ Institute of Physics, University of Tsukuba, Tsukuba, Ibaraki 305-8571, Japan \\ ${ }^{2}$ Institute for Cosmic Ray Research, University of Tokyo, Kashiwa, Chiba 277-8582, Japan \\ ${ }^{3}$ High Energy Accelerator Research Organization (KEK), Tsukuba, Ibaraki 305-0801, Japan \\ ${ }^{4}$ Center for Computational Physics, University of Tsukuba, Tsukuba, Ibaraki 305-8577, Japan \\ ${ }^{5}$ Department of Physics, Hiroshima University, Higashi-Hiroshima, Hiroshima 739-8526, Japan
}

(Received 2 June 2003; published 28 May 2004)

\begin{abstract}
We present a lattice QCD calculation of the heavy quark expansion parameters $\mu_{\pi}^{2}$ and $\mu_{G}^{2}$ for heavy-light mesons and heavy-light-light baryons. The calculation is carried out on a $20^{3} \times 48$ lattice at $\beta=6.0$ in the quenched approximation, using the lattice NRQCD action for heavy quarks. We obtain the parameters $\mu_{\pi}^{2}$ and $\mu_{G}^{2}$ in two different methods: a direct calculation of the matrix elements and an indirect calculation through the mass spectrum, and confirm that both the methods give consistent results. We also discuss an application to the lifetime ratios.
\end{abstract}

DOI: 10.1103/PhysRevD.69.094512

PACS number(s): $12.38 . \mathrm{Gc}, 12.39 . \mathrm{Hg}$

\section{INTRODUCTION}

The heavy quark expansion (HQE) $[1,2]$ is a fundamental tool in the study of heavy quark physics. The inclusive decay rate of heavy hadrons containing a single heavy quark may be expanded in terms of inverse heavy quark mass $1 / m_{Q}$ using the operator product expansion (OPE) technique, which enables us to calculate the inclusive rates in a model independent manner [3-6]. In particular, the determination of the Cabibbo-Kobayashi-Maskawa (CKM) matrix elements $\left|V_{c b}\right|$ and $\left|V_{u b}\right|$ through the corresponding semi-leptonic branching fractions relies on HQE.

It requires, however, several nonperturbative parameters as coefficients in HQE. At the order $1 / m_{Q}^{2}$ the nonperturbative parameters

$$
\begin{aligned}
& \mu_{\pi}^{2}\left(H_{Q}\right) \equiv \frac{1}{2 M_{H_{Q}}}\left\langle H_{Q}\left|\bar{Q}(i \vec{D})^{2} Q\right| H_{Q}\right\rangle, \\
& \mu_{G}^{2}\left(H_{Q}\right) \equiv \frac{1}{2 M_{H_{Q}}}\left\langle H_{Q}|\bar{Q} \vec{\sigma} \cdot \vec{B} Q| H_{Q}\right\rangle,
\end{aligned}
$$

appear in general. Here, $Q$ denotes a heavy quark field defined in the heavy quark effective theory (HQET), and $\left|H_{Q}\right\rangle$ represents a heavy-light meson or a heavy-light-light baryon state (for $b$ hadrons, $H_{b}=B, B^{*}, \Lambda_{b}, \Sigma_{b}, \Sigma_{b}^{*}$, etc.). Both parameters have mass dimension two, since they include a (spatial) covariant derivative squared $\vec{D}^{2}$ or a chromomagnetic operator $\vec{B}$. The inclusive decay rate of $H_{Q}$ is written in terms of $\mu_{\pi}^{2}\left(H_{Q}\right)$ and $\mu_{G}^{2}\left(H_{Q}\right)$ as

\footnotetext{
*Present address: Department of Physics, Hiroshima University, Higashi-Hiroshima, Hiroshima 739-8526, Japan.

†Present address: RIKEN BNL Research Center, Brookhaven National Laboratory, Upton, NY 11973.
}

$$
\begin{aligned}
\Gamma\left(H_{Q} \rightarrow X_{f}\right)= & \frac{G_{F}^{2} m_{Q}^{5}}{192 \pi^{3}}\left[c_{3}^{f}\left(1-\frac{\mu_{\pi}^{2}\left(H_{Q}\right)-\mu_{G}^{2}\left(H_{Q}\right)}{2 m_{Q}^{2}}\right)\right. \\
& \left.+2 c_{5}^{f} \frac{\mu_{G}^{2}\left(H_{Q}\right)}{m_{Q}^{2}}+\cdots\right],
\end{aligned}
$$

where the coefficients $c_{3}^{f}$ and $c_{5}^{f}$ are perturbatively calculable. On the other hand, the parameters $\mu_{\pi}^{2}\left(H_{Q}\right)$ and $\mu_{G}^{2}\left(H_{Q}\right)$ have to be extracted from some experimental data or calculated nonperturbatively. Several methods to determine $\mu_{\pi}^{2}$ and $\mu_{G}^{2}$ have been studied, and some of them are summarized in Sec. II.

In this work we calculate $\mu_{\pi}^{2}$ and $\mu_{G}^{2}$ in quenched lattice QCD using the NRQCD action including $O\left(1 / m_{Q}\right)$ terms for heavy quark. Since the matrix element of power divergent operator $\bar{Q}(i \vec{D})^{2} Q$ suffers from large perturbative uncertainty in the matching calculation with the continuum operator [7], we consider their difference between different hadron states, like $\mu_{\pi}^{2}\left(\Lambda_{b}\right)-\mu_{\pi}^{2}(B)$, in which the power divergence cancels. This kind of difference is also interesting in its own right, as it appears in the evaluation of lifetime difference of $b$ hadrons [8].

One of the advantages of this calculation is that we can choose several quark masses in the calculation so that the heavy quark mass dependence of the hadron masses and matrix elements may be studied. We calculate both matrix elements $\mu_{\pi}^{2}$ and $\mu_{G}^{2}$ and compare them with the corresponding mass spectrum and its heavy quark mass dependence. Another advantage in the use of the NRQCD lattice action is that the statistical signal in the Monte Carlo calculation is much better than in the static limit [9].

This paper is organized as follows. In Sec. II, the implications for the heavy quark expansion parameters from heavy hadron spectrum and the results of the previous nonperturbative calculations are discussed. In Sec. III we de- 
scribe our lattice calculation in detail. The results for hadron masses and heavy quark expansion parameters are shown in Sec. IV. The consistency check between the calculation of matrix elements and spectrum is also presented. Our results are applied to the lifetime ratio of different $b$ hadrons in Sec. $\mathrm{V}$. The conclusions are given in Sec. VI.

\section{HEAVY QUARK EXPANSION PARAMETERS}

In this section we briefly review the determination of the HQE parameters from mass spectrum and from some nonperturbative techniques. The determination through the measurements of several mass and energy moments in the inclusive $B \rightarrow X_{c} l \nu$ and $B \rightarrow X_{s} \gamma$ decays is another possibility [10-14], which is not covered in the following.

\section{A. Implications from spectroscopy}

The HQE parameters $\mu_{\pi}^{2}$ and $\mu_{G}^{2}$ defined in Eqs. (1) and (2) can be indirectly obtained through heavy hadron masses, using the HQE of hadron masses

$$
M_{H_{Q}}=m_{Q}+\bar{\Lambda}+\frac{\mu_{\pi}^{2}\left(H_{Q}\right)-\mu_{G}^{2}\left(H_{Q}\right)}{2 m_{Q}}+O\left(\frac{1}{m_{Q}^{2}}\right),
$$

where $\bar{\Lambda}$ is the residual energy difference between $M_{H_{Q}}$ and $m_{Q}$ surviving in the infinite heavy quark mass limit. The parameters $\mu_{\pi}^{2}$ and $\mu_{G}^{2}$ appear in the correction term of $O\left(1 / m_{Q}\right)$. Considering proper mass differences, certain combinations of $\bar{\Lambda}, \mu_{\pi}^{2}$ and $\mu_{G}^{2}$ can be extracted as shown below.

The notation $\lambda_{1}$ and $\lambda_{2}$ is often used instead of $\mu_{\pi}^{2}$ and $\mu_{G}^{2}$ for $B$ and $B^{*}$ mesons in the literature. The relation between $\lambda_{1,2}$ and $\mu_{\pi, G}^{2}$ is given by

$$
\begin{aligned}
& \lambda_{1} \equiv-\mu_{\pi}^{2}(B)=-\mu_{\pi}^{2}\left(B^{*}\right), \\
& \lambda_{2} \equiv \frac{1}{3} \mu_{G}^{2}(B)=-\mu_{G}^{2}\left(B^{*}\right),
\end{aligned}
$$

and the HQE of meson masses in Eq. (4) becomes

$$
\begin{gathered}
M_{B}=m_{b}+\bar{\Lambda}-\frac{\lambda_{1}+3 \lambda_{2}}{2 m_{b}}+O\left(\frac{1}{m_{b}^{2}}\right), \\
M_{B *}=m_{b}+\bar{\Lambda}-\frac{\lambda_{1}-\lambda_{2}}{2 m_{b}}+O\left(\frac{1}{m_{b}^{2}}\right) .
\end{gathered}
$$

The parameter $\lambda_{2}$ may be evaluated through the hyperfine splitting of ground state $B$ mesons as

$$
M_{B *}-M_{B}\left(\simeq \frac{4 \lambda_{2}}{2 m_{b}}\right)=46 \mathrm{MeV},
$$

or, equivalently

$$
\lambda_{2} \simeq \frac{1}{4}\left(M_{B *}^{2}-M_{B}^{2}\right)=0.12 \mathrm{GeV}^{2},
$$

at the leading order.

For $\Lambda_{b}$ baryon, the parameter $\mu_{G}^{2}\left(\Lambda_{b}\right)$ vanishes, since the light degrees of freedom is spin singlet inside $\Lambda_{b}$. The relations

$$
\begin{gathered}
\mu_{\pi}^{2}\left(\Sigma_{b}\right)=\mu_{\pi}^{2}\left(\Sigma_{b}^{*}\right), \\
\frac{1}{2} \mu_{G}^{2}\left(\Sigma_{b}\right)=-\mu_{G}^{2}\left(\Sigma_{b}^{*}\right),
\end{gathered}
$$

hold for $\Sigma_{b}$ and $\Sigma_{b}^{*}$ baryons, as they are related by spin rotations, analogous to Eqs. (5) and (6) for $B^{(*)}$ mesons.

The spin-averaged meson mass becomes independent of $\lambda_{2}$

$$
M_{\bar{B}} \equiv \frac{M_{B}+3 M_{B^{*}}}{4}=m_{b}+\bar{\Lambda}-\frac{\lambda_{1}}{2 m_{b}}+O\left(\frac{1}{m_{b}^{2}}\right),
$$

but $\lambda_{1}$ cannot be extracted solely from this expression, as it appears together with the lowest order parameter $\bar{\Lambda}$. In order to proceed further, we have to consider a similar relation for the $D$ meson and take a mass difference to obtain

$$
M_{\bar{B}}-M_{\bar{D}}=m_{b}-m_{c}-\lambda_{1}\left(\frac{1}{2 m_{b}}-\frac{1}{2 m_{c}}\right)+O\left(\frac{1}{m_{b, c}^{2}}\right) \text {. }
$$

The leading dependence on the heavy quark masses $m_{b}$ and $m_{c}$ can be subtracted out if we take a double mass difference

$$
\mu_{\pi}^{2}\left(\Lambda_{b}\right)-\mu_{\pi}^{2}(B)=2 \frac{\left(M_{\Lambda_{b}}-M_{\Lambda_{c}}\right)-\left(M_{\bar{B}}-M_{\bar{D}}\right)}{\frac{1}{M_{\bar{B}}}-\frac{1}{M_{\bar{D}}}}+O\left(\frac{1}{m_{b, c}}\right)
$$

from which we obtain

$$
\mu_{\pi}^{2}\left(\Lambda_{b}\right)-\mu_{\pi}^{2}(B)=-0.01 \pm 0.03 \mathrm{GeV}^{2} .
$$

This argument relies on HQE truncated at order $1 / m_{Q}$, which is questionable for charmed mesons and baryons. Therefore, for the use of the HQE parameter $\mu_{\pi}^{2}$ in other phenomenological analysis, some independent theoretical calculations are desirable.

\section{B. Nonperturbative calculations}

The determination of $\lambda_{1}$ using the QCD sum rule has been attempted by two groups and reached conflicting results $\lambda_{1}=-0.5 \pm 0.2 \mathrm{GeV}^{2} \quad[15]$ and $-0.1 \pm 0.05 \mathrm{GeV}^{2}$ [16]. Their difference is explained to come from non-diagonal matrix elements like $\left\langle B\left|\bar{Q}(i \vec{D})^{2} Q\right| B^{\prime}\right\rangle$, where $B^{\prime}$ is an excited state of $B$ meson [2]. Since there is no definite way to evaluate these matrix elements at present, it is not straightforward to improve the determination of $\lambda_{1}$ within the QCD sum rule technique.

The lattice QCD can also be used to determine the HQE parameters. In the lattice calculation of the matrix element 
TABLE I. Simulation parameters. The parameters $a$ and $b$ are for the smearing function $e^{-a \cdot r^{b}}$.

\begin{tabular}{lccccc}
\hline \hline$a M_{0}$ & 1.3 & 2.1 & 3.0 & 5.0 & 10.0 \\
\hline$n$ & 3 & 3 & 2 & 2 & 2 \\
$a$ & 0.2248 & 0.2530 & 0.2711 & 0.3074 & 0.3425 \\
$b$ & 1.2484 & 1.1840 & 1.1465 & 1.0794 & 1.0294 \\
\hline \hline
\end{tabular}

$\left\langle B\left|\bar{Q}(i \vec{D})^{2} Q\right| B\right\rangle$ the subtraction of quadratic divergence is essential, since otherwise the perturbative expansion to relate lattice and continuum operators poorly converges [7]. First lattice calculation with such nonperturbative subtraction was done by Crisafulli et al. [17] using the HQET on the lattice, which was updated in Gimenez et al. [18], and the result is $\lambda_{1}=0.09 \pm 0.14 \mathrm{GeV}^{2}$.

Another possible approach on the lattice is to fit the measured mass spectrum for various heavy quark masses with the mass relation (4). Ali Khan et al. [19] performed such analysis for $b$ flavored mesons and baryons using the lattice NRQCD for heavy quark. Their result is $\lambda_{1}=-0.1$ $\pm 0.4 \mathrm{GeV}^{2}$ for $B$ meson. Kronfeld and Simone [20] performed similar analysis with a larger set of lattice data of heavy-light mesons, and quoted $\lambda_{1}=-0.45 \pm 0.12 \mathrm{GeV}^{2}$. The calculation of $\mu_{\pi}^{2}$ for $b$ baryon is available only from Ali Khan et al. [19]. They quoted $\mu_{\pi}^{2}\left(\Lambda_{b}\right)=-1.7 \pm 3.4 \mathrm{GeV}^{2}$.

For the parameter $\lambda_{2}$, Gimenez et al. [18] found $\lambda_{2}$ $=0.07 \pm 0.01 \mathrm{GeV}^{2}$ from the direct calculation of the matrix element. Ali Khan et al. [19] estimated $\lambda_{2}\left(B_{d}\right)=0.069$ $\pm 0.019 \mathrm{GeV}^{2}$ and $\lambda_{2}\left(B_{s}\right)=0.078 \pm 0.012 \mathrm{GeV}^{2}$ from the hyperfine splitting measured on the lattice.

The difference of $\bar{\Lambda}$ between several heavy hadrons is only estimated from the mass difference. Ali Khan et al. [19] estimated $\bar{\Lambda}\left(\Lambda_{b}\right)-\bar{\Lambda}(B)=415 \pm 156 \mathrm{MeV}, \bar{\Lambda}\left(\Sigma_{b}\right)-\bar{\Lambda}\left(\Lambda_{b}\right)$ $=176 \pm 152 \mathrm{MeV}$ and $\bar{\Lambda}\left(B_{s}\right)-\bar{\Lambda}\left(B_{d}\right)=81 \pm 31 \mathrm{MeV}$.

In this work we calculate $\mu_{\pi}^{2}$ and $\mu_{G}^{2}$ on the lattice for ground state mesons and baryons. We use the both methods, namely the direct measurement of the matrix elements and the extraction from the heavy hadron spectrum. The difference of $\bar{\Lambda}$ is also evaluated from the mass difference.

\section{LATTICE CALCULATION}

In this section we present the details of our lattice calculation, which include the definition of the NRQCD action, simulation parameters, and the method to extract the matrix elements. The matching of lattice operators onto their continuum counterpart is also discussed.

\section{A. Lattice NRQCD}

We use the lattice NRQCD action [21,22] for heavy quark. The particular form of the action used in this work is the same as in $[23,24]$

$$
S_{\mathrm{NRQCD}}=\sum_{x, y} Q^{\dagger}(x)\left[\delta_{x, y}-K_{Q}(x, y)\right] Q(y) .
$$

TABLE II. Perturbative coefficients appearing in the calculation of hadron masses from Eq. (38). The perturbative expansion is given as $a E_{0}=\alpha_{s} A$ and $Z_{m}=1+\alpha_{s} B$ where the coefficients $A$ and $B$ are given in [23]. For the numerical analysis we use a renormalized coupling $\alpha_{V}(1 / a)=0.256$ for $\alpha_{s}$ at $\beta=6.0 . a \Delta$ in the last column is defined as $a \Delta=Z_{m} a M_{0}-a E_{0}$.

\begin{tabular}{lcccccc}
\hline \hline$a M_{0}$ & $n$ & $A$ & $B$ & $a E_{0}$ & $Z_{m}$ & $a \Delta$ \\
\hline 1.3 & 3 & 0.547 & 0.914 & 0.140 & 1.234 & 1.464 \\
2.1 & 3 & 0.754 & 0.578 & 0.193 & 1.148 & 2.218 \\
3.0 & 2 & 0.855 & 0.381 & 0.219 & 1.097 & 3.072 \\
5.0 & 2 & 0.946 & 0.176 & 0.242 & 1.045 & 4.983 \\
10.0 & 2 & 1.011 & 0.040 & 0.259 & 1.010 & 9.841 \\
\hline \hline
\end{tabular}

The kernel to describe the time evolution of heavy quark is given by

$$
\begin{aligned}
K_{Q}(x, y) \equiv & \left(1-\frac{a H_{0}}{2 n}\right)_{t+1}^{n}\left(1-\frac{a \delta H}{2}\right)_{t+1} \delta_{4}^{(-)} U_{4}^{\dagger}(t) \\
& \times\left(1-\frac{a \delta H}{2}\right)_{t}\left(1-\frac{a H_{0}}{2 n}\right)_{t}^{n},
\end{aligned}
$$

where the index to label the spatial coordinate is suppressed. The operator $\delta_{4}^{(-)}$is defined as $\delta_{4}^{(-)}(x, y) \equiv \delta_{x_{4}-1, y_{4}} \delta_{\vec{x}, \vec{y}}$, and

$$
\begin{aligned}
& H_{0} \equiv-\frac{\Delta^{(2)}}{2 m_{Q}}, \\
& \delta H \equiv-c_{B} \frac{g}{2 m_{Q}} \vec{\sigma} \cdot \vec{B} .
\end{aligned}
$$

$\Delta^{(2)}$ is a lattice covariant Laplacian

$$
\begin{aligned}
\Delta^{(2)} Q(x)= & \sum_{i=1}^{3} \Delta_{i}^{(2)} Q(x) \\
= & \sum_{i=1}^{3}\left[U_{i}(x) Q(x+\hat{i})\right. \\
& \left.+U_{i}^{\dagger}(x-\hat{i}) Q(x-\hat{i})-2 Q(x)\right],
\end{aligned}
$$

and the chromomagnetic field $\vec{B}$ is defined as the clover-leaf type on the lattice [22]. The parameter $n$ in the evolution kernel (18) is a positive integer introduced to stabilize unphysical momentum modes [21,22]. With these definitions the lattice NRQCD action (17) deduces to the usual continuum NRQCD action

$$
\mathcal{L}_{\mathrm{NRQCD}}^{\text {cont }}=Q^{\dagger}\left[D_{0}+\frac{\vec{D}^{2}}{2 M}+g \frac{\vec{\sigma} \cdot \vec{B}}{2 M}\right] Q
$$

in the limit of vanishing lattice spacing.

The parameters appearing in the NRQCD action (17), $m_{Q}$ and $c_{B}$ at this order, have to be matched onto their continuum counterparts using perturbation theory. The matching of heavy quark mass $m_{Q}$ is done through the calculation of 

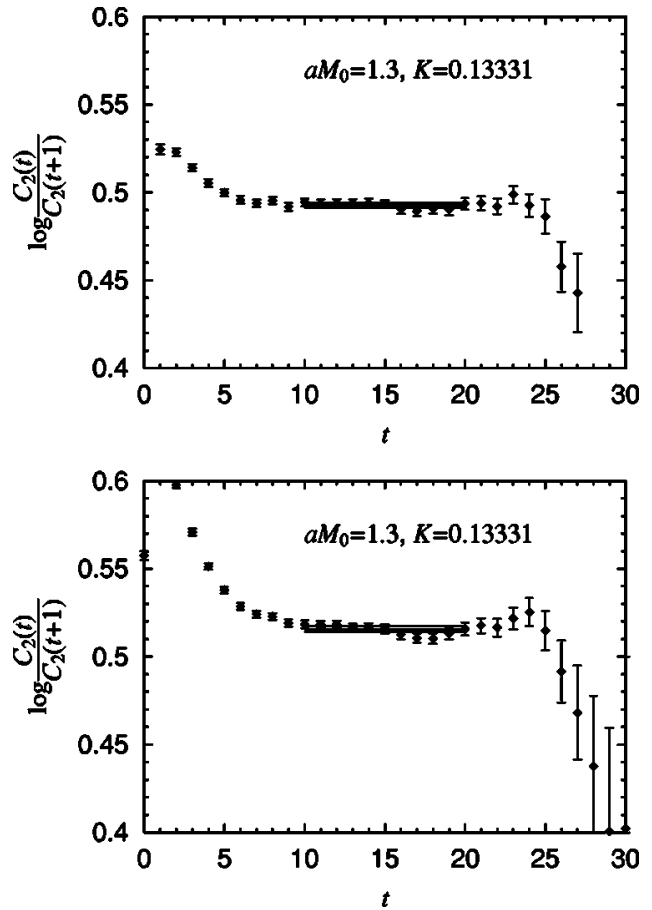

FIG. 1. Effective mass plot for the $B$ (top panel) and $B^{*}$ (bottom panel) mesons at $K=0.13331$ and $a M_{0}=1.3$. Solid lines represent the fitting result with an error band of one standard deviation.

hadron masses as described later. On the other hand, the one-loop calculation for $c_{B}$ is unfortunately not yet available, so we use the tree level value $c_{B}=1$. However, we apply the mean field improvement of the gauge link variable $U_{\mu}(x)$ $\rightarrow U_{\mu}(x) / u_{0}[25]$ everywhere it appears, with $u_{0}$ a mean link value defined through the plaquette expectation value $u_{0}$ $\equiv\left\langle\frac{1}{3} \operatorname{Tr} U_{P}\right\rangle$. With the mean field improvement we expect that the tree level matching is reasonably good. Furthermore, the final predictions for the matrix elements deduced from our analysis are given in the static limit, which is irrelevant to the parameter $c_{B}$.

The four-component heavy quark field $h$ used to construct the hadron interpolating fields is related to the twocomponent nonrelativistic field $Q$ through the FoldyWouthuysen-Tani (FWT) transformation

$$
h=R\left(\begin{array}{l}
Q \\
0
\end{array}\right),
$$

with the rotation matrix $R$ given by

$$
R=1-\frac{\vec{\gamma} \cdot \vec{\Delta}}{2 m_{Q}}
$$

at order $1 / m_{Q}$. Our convention for the gamma matrices is

$$
\gamma_{4}=\left(\begin{array}{cc}
\mathrm{I} & 0 \\
0 & -\mathrm{I}
\end{array}\right), \quad \vec{\gamma}=\left(\begin{array}{cc}
0 & -i \vec{\sigma} \\
i \vec{\sigma} & 0
\end{array}\right)
$$

and the spatial covariant derivative is defined as
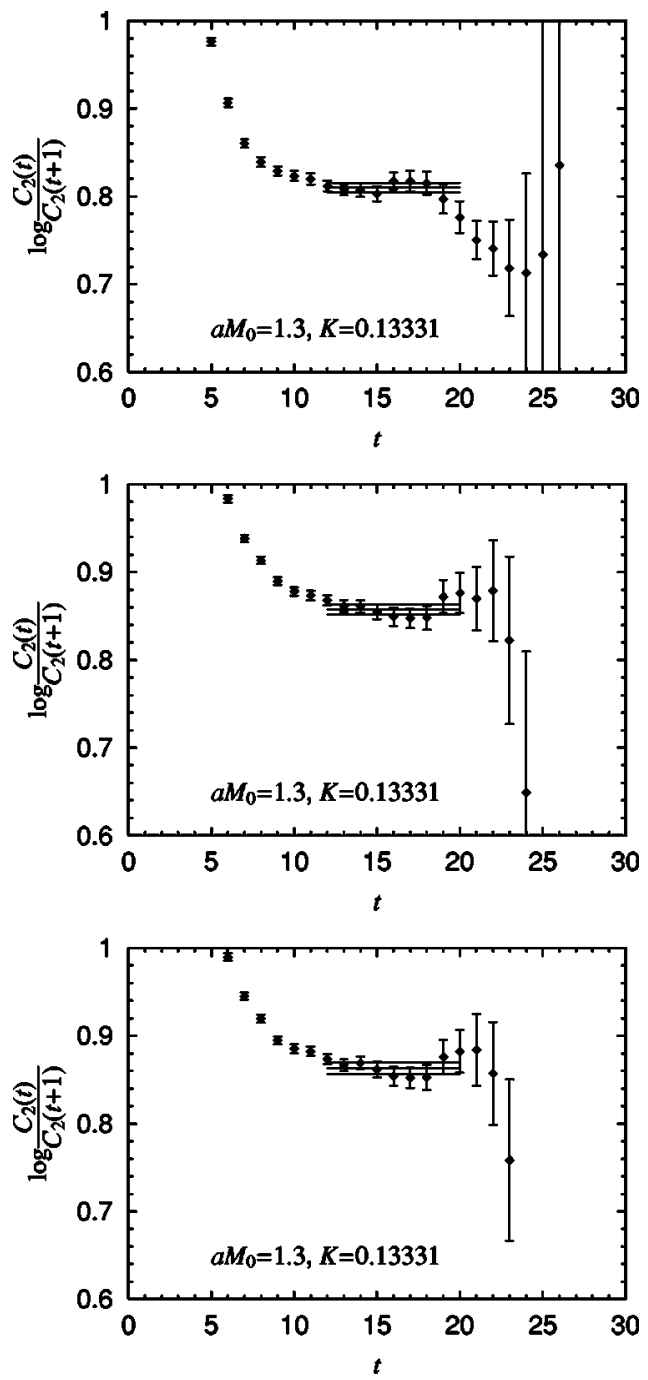

FIG. 2. Effective mass plot for the $\Lambda_{b}$ (top panel) and $\Sigma_{b}$ (middle panel) and $\Sigma_{b}^{*}$ (bottom panel) baryons at $K=0.13331$ and $a M_{0}=1.3$. Solid lines represent the fitting result with an error band of one standard deviation.

$$
\Delta_{i} Q(x)=\frac{1}{2}\left[U_{i}(x) Q(x+\hat{i})-U_{i}^{\dagger}(x-\hat{i}) Q(x-\hat{i})\right] .
$$

\section{B. Simulation details}

Our calculation is carried out in quenched lattice QCD at $\beta=6.0$ on a $20^{3} \times 48$ lattice. Gauge configurations are generated with the single plaquette action, and 515 configurations are analyzed.

The NRQCD action including $O\left(1 / m_{Q}\right)$ described in the previous subsection is adapted for heavy quarks. Five heavy quark masses $a m_{Q}=1.3,2.1,3.0,5.0$, and 10.0 are simulated to study the $m_{Q}$ dependence of hadron masses and matrix elements. The details on the parameters for heavy quark are shown in Table I.

For light quarks, the $O(a)$-improved Wilson action [26] with the nonperturbatively tuned coefficient $c_{\mathrm{SW}}=1.769$ [27] is used. Three hopping parameters $K=0.13331,0.13384$, and 0.13432 are employed to extrapolate to the chiral 
TABLE III. Binding energy of heavy-light mesons and heavy-light-light baryons.

\begin{tabular}{|c|c|c|c|c|c|c|}
\hline$a M_{0}$ & $K$ & $a E_{\text {sim }}(B)$ & $a E_{s i m}\left(B^{*}\right)$ & $a E_{s i m}\left(\Lambda_{b}\right)$ & $a E_{\text {sim }}\left(\Sigma_{b}\right)$ & $a E_{s i m}\left(\Sigma_{b}^{*}\right)$ \\
\hline 1.3 & \multirow[t]{5}{*}{0.13331} & $0.4928(15)$ & $0.5156(17)$ & $0.8101(54)$ & $0.8573(60)$ & $0.8631(63)$ \\
\hline 2.1 & & $0.5145(17)$ & $0.5298(19)$ & $0.8256(60)$ & $0.8725(65)$ & $0.8766(67)$ \\
\hline 3.0 & & $0.5247(19)$ & $0.5357(21)$ & $0.8324(66)$ & $0.8787(70)$ & $0.8816(72)$ \\
\hline 5.0 & & $0.5327(24)$ & $0.5391(25)$ & $0.8386(92)$ & $0.8830(84)$ & $0.8843(86)$ \\
\hline 10.0 & & $0.5376(37)$ & $0.5401(38)$ & $0.847(16)$ & $0.891(14)$ & $0.891(14)$ \\
\hline 1.3 & \multirow[t]{5}{*}{0.13384} & $0.4754(18)$ & $0.4987(20)$ & $0.7680(73)$ & $0.8210(85)$ & $0.8275(91)$ \\
\hline 2.1 & & $0.4976(21)$ & $0.5132(22)$ & $0.7849(83)$ & $0.8366(92)$ & $0.8411(97)$ \\
\hline 3.0 & & $0.5083(23)$ & $0.5194(25)$ & $0.7927(95)$ & $0.843(10)$ & $0.847(10)$ \\
\hline 5.0 & & $0.5166(29)$ & $0.5229(30)$ & $0.801(12)$ & $0.850(12)$ & $0.852(12)$ \\
\hline 10.0 & & $0.5218(43)$ & $0.5241(44)$ & $0.813(21)$ & $0.862(19)$ & $0.862(19)$ \\
\hline 1.3 & \multirow[t]{5}{*}{0.13432} & $0.4599(24)$ & $0.4836(26)$ & $0.728(12)$ & $0.786(15)$ & $0.793(15)$ \\
\hline 2.1 & & $0.4825(27)$ & $0.4983(29)$ & $0.746(14)$ & $0.801(16)$ & $0.806(16)$ \\
\hline 3.0 & & $0.4934(30)$ & $0.5047(32)$ & $0.756(15)$ & $0.808(17)$ & $0.812(17)$ \\
\hline 5.0 & & $0.5021(36)$ & $0.5085(38)$ & $0.767(19)$ & $0.820(20)$ & $0.821(20)$ \\
\hline 10.0 & & $0.5079(57)$ & $0.5099(55)$ & $0.782(32)$ & $0.831(24)$ & $0.830(23)$ \\
\hline 1.3 & \multirow[t]{5}{*}{$K_{s}$} & $0.4826(17)$ & $0.5057(19)$ & $0.7850(66)$ & $0.8357(78)$ & $0.8419(82)$ \\
\hline 2.1 & & $0.5046(20)$ & $0.5201(21)$ & $0.8015(75)$ & $0.8510(83)$ & $0.8553(87)$ \\
\hline 3.0 & & $0.5151(22)$ & $0.5262(23)$ & $0.8089(84)$ & $0.8575(90)$ & $0.8606(93)$ \\
\hline 5.0 & & $0.5233(27)$ & $0.5296(28)$ & $0.816(11)$ & $0.864(11)$ & $0.865(11)$ \\
\hline 10.0 & & $0.5284(41)$ & $0.5307(42)$ & $0.827(18)$ & $0.873(16)$ & $0.873(16)$ \\
\hline 1.3 & \multirow[t]{5}{*}{$K_{c}$} & $0.4290(33)$ & $0.4534(36)$ & $0.652(17)$ & $0.721(21)$ & $0.729(22)$ \\
\hline 2.1 & & $0.4524(37)$ & $0.4688(39)$ & $0.673(20)$ & $0.736(23)$ & $0.742(24)$ \\
\hline 3.0 & & $0.4639(42)$ & $0.4756(43)$ & $0.684(23)$ & $0.744(24)$ & $0.749(25)$ \\
\hline 5.0 & & $0.4733(48)$ & $0.4796(52)$ & $0.699(28)$ & $0.761(29)$ & $0.762(29)$ \\
\hline 10.0 & & $0.4799(77)$ & $0.4814(74)$ & $0.722(49)$ & $0.777(36)$ & $0.776(34)$ \\
\hline
\end{tabular}
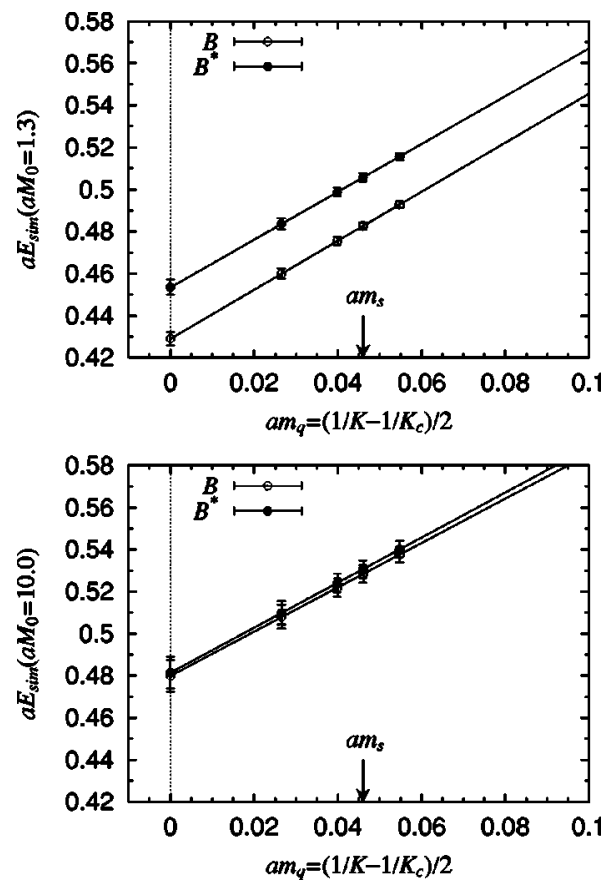

FIG. 3. Binding energy of the $B$ and $B^{*}$ mesons as a function of light quark mass at $a M_{0}=1.3$ (top panel) and 10.0 (bottom panel).
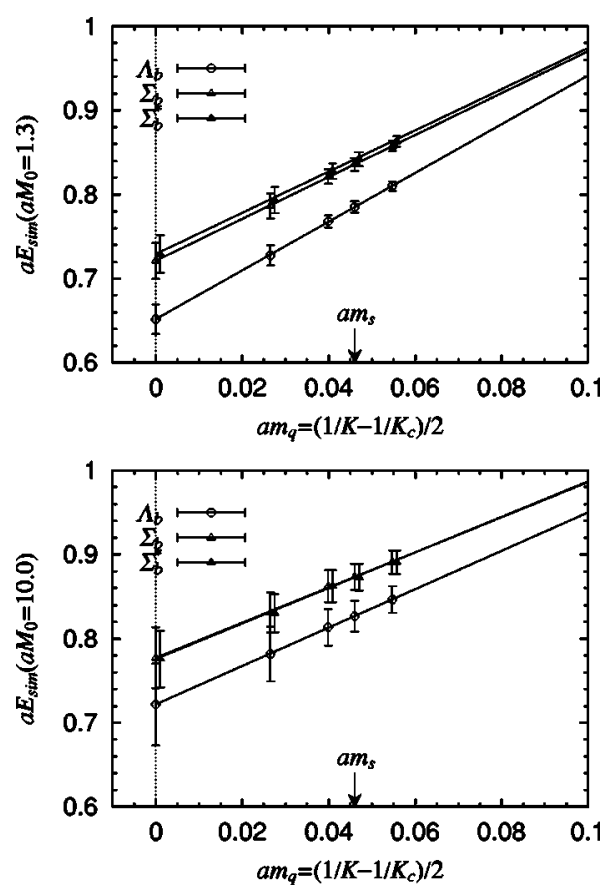

FIG. 4. Binding energy for the $\Lambda_{b}, \Sigma_{b}$ and $\Sigma_{b}^{*}$ baryons as a function of light quark mass at $a M_{0}=1.3$ (top panel) and 10.0 (bottom panel). 

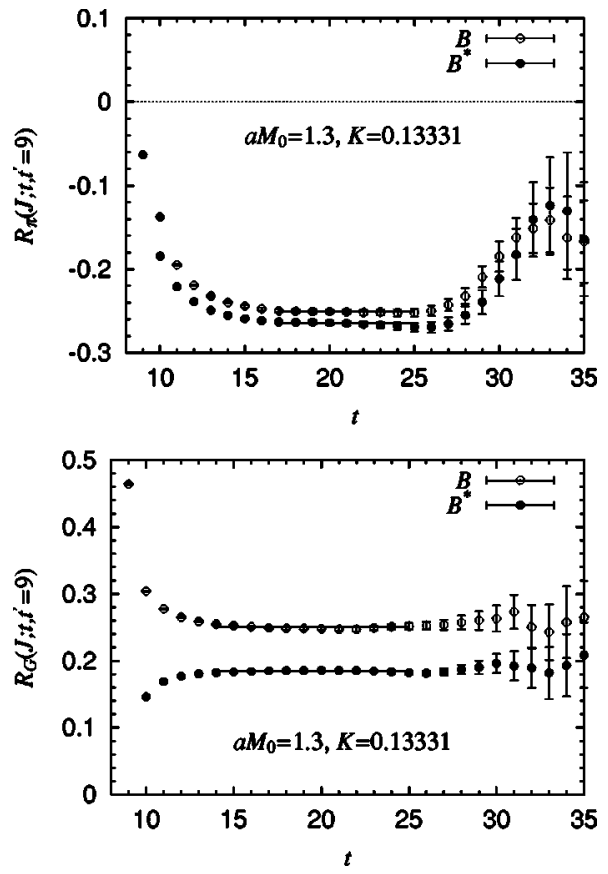

FIG. 5. Ratio $R_{i}\left(J ; t, t^{\prime}=9\right.$ ) for $\mu_{\pi}^{2}$ (top panel) and for $\mu_{G}^{2}$ (bottom panel) at $K=0.13331$ and $a M=1.3$. Open (filled) symbols are the data for the $B\left(B^{*}\right)$ meson. Solid lines represent a constant fit with an fit interval $[17,25]$ for $\mu_{\pi}^{2}$ or $[14,25]$ for $\mu_{G}^{2}$.

limit $K_{c}=0.135284(8)$. The inverse lattice spacing $a^{-1}$ $=1.85(5) \mathrm{GeV}$ is determined through the $\rho$ meson mass $m_{\rho}=770 \mathrm{MeV}$. The strange quark mass $a m_{s}=0.0460(22)$ is fixed using $m_{K} / m_{\rho}=0.644$ as an input. These values are consistent with other works using the same $\beta$ and $c_{\text {SW }}$ values. Other input parameters may yield different results for the lattice spacing, which is attributed to the error due to the quenched approximation. The quenching error may arise for other quantities calculated in this paper, but the estimation of the quenching effect is not given as it is beyond the scope of this paper.

\section{Hadron masses}

The hadron masses are measured through the asymptotic behavior of two-point functions

$$
C(J ; t)=\sum_{\vec{x}}\left\langle J(\vec{x}, t) J^{(S) \dagger}(\overrightarrow{0}, 0)\right\rangle \rightarrow e^{-E_{s i m} t}
$$

for sufficiently large time separation $t$. With the NRQCD action, for which the bare heavy quark mass is subtracted from the formulation, we obtain the binding energy $E_{\text {sim }}$ from the two-point function. The interpolating operator $J$ is chosen such that it shares the same quantum number with the hadron of interest. The hadrons and their interpolating operators we consider in this work are the following:

$$
\begin{aligned}
B & =\bar{d} \gamma_{4} \gamma_{5} h, \\
B^{*} & =\bar{d} \gamma_{i} h,
\end{aligned}
$$
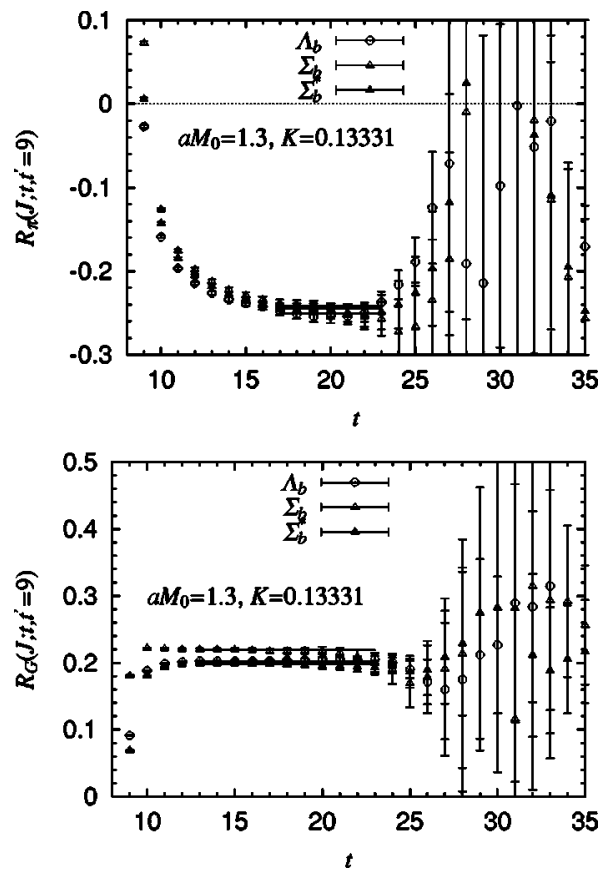

FIG. 6. Ratio $R_{i}\left(J ; t, t^{\prime}=9\right)$ for $\mu_{\pi}^{2}$ (top panel) and for $\mu_{G}^{2}$ (bottom panel) at $K=0.13331$ and $a M=1.3$. Open circles, open triangles and filled triangles are data for $\Lambda_{b}, \Sigma_{b}$ and $\Sigma_{b}^{*}$ baryons, respectively. Solid lines represent a constant fit with an fit interval $[17,23]$ for $\mu_{\pi}^{2}$ or $[13,23]$ for $\mu_{G}^{2}$.

$$
\begin{gathered}
\Lambda_{b}\left(s_{z}=+1 / 2\right)=\epsilon_{a b c}\left(u^{a} C \gamma_{5} d^{b}\right) h_{\uparrow}^{c}, \\
\Lambda_{b}\left(s_{z}=-1 / 2\right)=\epsilon_{a b c}\left(u^{a} C \gamma_{5} d^{b}\right) h_{\downarrow}^{c}, \\
\Sigma_{b}\left(s_{z}=+1 / 2\right)=-\frac{1}{\sqrt{3}} \epsilon_{a b c}\left(u^{a} C \gamma_{3} d^{b}\right) h_{\uparrow}^{c} \\
+\sqrt{\frac{2}{3}} \epsilon_{a b c}\left(u^{a} C \frac{\gamma_{1}-i \gamma_{2}}{2} d^{b}\right) h_{\downarrow}^{c},
\end{gathered}
$$

$$
\begin{aligned}
\Sigma_{b}\left(s_{z}=\right. & -1 / 2)=-\sqrt{\frac{2}{3}} \epsilon_{a b c}\left(u^{a} C \frac{\gamma_{1}+i \gamma_{2}}{2} d^{b}\right) h_{\uparrow}^{c} \\
& +\frac{1}{\sqrt{3}} \epsilon_{a b c}\left(u^{a} C \gamma_{3} d^{b}\right) h_{\downarrow}^{c},
\end{aligned}
$$

$$
\Sigma_{b}^{*}\left(s_{z}=+3 / 2\right)=\epsilon_{a b c}\left(u^{a} C \frac{\gamma_{1}-i \gamma_{2}}{2} d^{b}\right) h_{\uparrow}^{c},
$$

$$
\begin{aligned}
\Sigma_{b}^{*}\left(s_{z}=\right. & +1 / 2)=\sqrt{\frac{2}{3}} \epsilon_{a b c}\left(u^{a} C \gamma_{3} d^{b}\right) h_{\uparrow}^{c} \\
& +\frac{1}{\sqrt{3}} \epsilon_{a b c}\left(u^{a} C \frac{\gamma_{1}-i \gamma_{2}}{2} d^{b}\right) h_{\downarrow}^{c},
\end{aligned}
$$


TABLE IV. Matrix element $\mu_{\pi}^{2}$ for heavy-light mesons and heavy-light-light baryons.

\begin{tabular}{|c|c|c|c|c|c|c|}
\hline$a M_{0}$ & $K$ & $a^{2} \mu_{\pi}^{2}(B)$ & $a^{2} \mu_{\pi}^{2}\left(B^{*}\right)$ & $a^{2} \mu_{\pi}^{2}\left(\Lambda_{b}\right)$ & $a^{2} \mu_{\pi}^{2}\left(\Sigma_{b}\right)$ & $a^{2} \mu_{\pi}^{2}\left(\Sigma_{b}^{*}\right)$ \\
\hline 1.3 & 0.13331 & $-0.2507(24)$ & $-0.2643(25)$ & $-0.2503(62)$ & $-0.2416(56)$ & $-0.2449(55)$ \\
\hline 2.1 & & $-0.0998(51)$ & $-0.1075(53)$ & $-0.096(15)$ & $-0.083(11)$ & $-0.085(11)$ \\
\hline 3.0 & & $-0.0546(90)$ & $-0.0558(94)$ & $-0.051(27)$ & $-0.036(19)$ & $-0.037(19)$ \\
\hline 5.0 & & $-0.016(23)$ & $-0.003(24)$ & $-0.072(63)$ & $-0.053(52)$ & $-0.048(52)$ \\
\hline 10.0 & & $-0.030(88)$ & $+0.018(88)$ & $-0.28(27)$ & $-0.35(25)$ & $-0.34(25)$ \\
\hline 1.3 & 0.13384 & $-0.2525(30)$ & $-0.2663(30)$ & $-0.2545(81)$ & $-0.2416(82)$ & $-0.2427(82)$ \\
\hline 2.1 & & $-0.1027(63)$ & $-0.1104(66)$ & $-0.101(19)$ & $-0.081(17)$ & $-0.081(16)$ \\
\hline 3.0 & & $-0.059(11)$ & $-0.059(12)$ & $-0.058(36)$ & $-0.035(28)$ & $-0.036(28)$ \\
\hline 5.0 & & $-0.022(28)$ & $-0.003(29)$ & $-0.103(89)$ & $-0.082(77)$ & $-0.076(79)$ \\
\hline 10.0 & & $-0.05(11)$ & $+0.01(11)$ & $-0.39(39)$ & $-0.51(38)$ & $-0.52(39)$ \\
\hline 1.3 & 0.13432 & $-0.2537(40)$ & $-0.2678(40)$ & $-0.257(13)$ & $-0.229(14)$ & $-0.225(14)$ \\
\hline 2.1 & & $-0.1042(84)$ & $-0.1128(88)$ & $-0.099(31)$ & $-0.057(27)$ & $-0.054(26)$ \\
\hline 3.0 & & $-0.062(15)$ & $-0.062(16)$ & $-0.049(58)$ & $-0.008(47)$ & $-0.005(47)$ \\
\hline 5.0 & & $-0.029(37)$ & $-0.004(38)$ & $-0.13(15)$ & $-0.10(13)$ & $-0.09(14)$ \\
\hline 10.0 & & $-0.09(14)$ & $+0.00(14)$ & $-0.45(66)$ & $-0.71(69)$ & $-0.76(70)$ \\
\hline 1.3 & $K_{s}$ & $-0.2517(28)$ & $-0.2654(28)$ & $-0.2526(74)$ & $-0.2399(73)$ & $-0.2415(72)$ \\
\hline 2.1 & & $-0.1014(58)$ & $-0.1092(61)$ & $-0.098(18)$ & $-0.078(14)$ & $-0.079(14)$ \\
\hline 3.0 & & $-0.057(10)$ & $-0.058(11)$ & $-0.053(33)$ & $-0.032(25)$ & $-0.032(25)$ \\
\hline 5.0 & & $-0.020(26)$ & $-0.003(27)$ & $-0.090(80)$ & $-0.069(68)$ & $-0.063(70)$ \\
\hline 10.0 & & $-0.05(10)$ & $+0.01(10)$ & $-0.34(34)$ & $-0.45(34)$ & $-0.46(34)$ \\
\hline 1.3 & $K_{c}$ & $-0.2567(55)$ & $-0.2712(55)$ & $-0.265(19)$ & $-0.227(20)$ & $-0.218(20)$ \\
\hline 2.1 & & $-0.109(12)$ & $-0.118(12)$ & $-0.107(44)$ & $-0.049(40)$ & $-0.041(40)$ \\
\hline 3.0 & & $-0.069(21)$ & $-0.067(21)$ & $-0.057(82)$ & $-0.001(70)$ & $+0.004(71)$ \\
\hline 5.0 & & $-0.040(51)$ & $-0.006(52)$ & $-0.19(22)$ & $-0.15(20)$ & $-0.14(21)$ \\
\hline 10.0 & & $-0.14(20)$ & $-0.01(20)$ & $-0.64(96)$ & $-1.01(98)$ & $-1.1(1.0)$ \\
\hline
\end{tabular}

$$
\begin{aligned}
& \Sigma_{b}^{*}\left(s_{z}=\right.-1 / 2)=\frac{1}{\sqrt{3}} \epsilon_{a b c}\left(u^{a} C \frac{\gamma_{1}+i \gamma_{2}}{2} d^{b}\right) h_{\uparrow}^{c} \\
&+\sqrt{\frac{2}{3}} \epsilon_{a b c}\left(u^{a} C \gamma_{3} d^{b}\right) h_{\downarrow}^{c}, \\
& \Sigma_{b}^{*}\left(s_{z}=-3 / 2\right)=\epsilon_{a b c}\left(u^{a} C \frac{\gamma_{1}+i \gamma_{2}}{2} d^{b}\right) h_{\uparrow}^{c} .
\end{aligned}
$$

Although the notations motivated from the $b$ hadron spectrum are used, we use them for general heavy quark mass we consider. The light quark fields $u$ and $d$ denote the relativistic up and down quark fields, respectively. The heavy quark field $h$ has a subscript $\uparrow$ or $\downarrow$, which represents its spin component in the $z$ direction. We assume the Dirac representation of gamma matrices, and $s_{z}$ means the $z$ component of the spin of baryons. The charge conjugation matrix $C$ has a representation $C=\gamma_{0} \gamma_{2}$. The superscript $a, b$ or $c$ denotes a color index of quarks.

The smeared operator $J^{(S)}$ is used at the source in Eq. (27) to enhance the overlap with the ground state. It is defined such that the heavy quark field is smeared according to an exponential form $e^{-a \cdot r^{b}}$ around the light quark field fixed at the origin. $r$ is a distance from the origin, and the parameters $a$ and $b$ are measured for the pion wave function. Thus, they depend on the light quark mass, as listed in Table I. Although it is not an optimal choice for heavy hadrons, the plateau is satisfactory as we demonstrate later.

The hadron mass is obtained through the relation

$$
M_{\mathrm{had}}=\left(Z_{m} M_{0}-E_{0}\right)+E_{\mathrm{sim}},
$$

where $Z_{m}$ is the mass renormalization factor which relates the bare quark mass $M_{0}$ with the pole mass and $E_{0}$ is the energy shift of the heavy quark. These factors are perturbatively calculated at the one-loop level in [23] for our choice of heavy quark action. We summarize them in Table II.

\section{Matrix elements}

To calculate the expansion parameters $\mu_{\pi}^{2}$ and $\mu_{G}^{2}$ from three-point functions, we construct a ratio

$$
R_{i}\left(J ; t, t^{\prime}\right)=\frac{\sum_{\vec{x}, \vec{y}}\left\langle J(\vec{x}, t) O_{i}\left(\vec{y}, t^{\prime}\right) J^{(S) \dagger}(\overrightarrow{0}, 0)\right\rangle}{\sum_{\vec{x}}\left\langle J(\vec{x}, t) J^{(S) \dagger}(\overrightarrow{0}, 0)\right\rangle}
$$

with $O_{i}$ either the kinetic operator

$$
O_{\pi}=\bar{Q}(i \vec{D})^{2} Q
$$

or the chromomagnetic operator 
TABLE V. Matrix element $\mu_{G}^{2}$ for heavy-light mesons and heavy-light-light baryons.

\begin{tabular}{|c|c|c|c|c|c|c|}
\hline$a M_{0}$ & $K$ & $a^{2} \mu_{G}^{2}(B)$ & $a^{2} \mu_{G}^{2}\left(B^{*}\right)$ & $a^{2} \mu_{G}^{2}\left(\Lambda_{b}\right)$ & $a^{2} \mu_{G}^{2}\left(\Sigma_{b}\right)$ & $a^{2} \mu_{G}^{2}\left(\Sigma_{b}^{*}\right)$ \\
\hline 1.3 & \multirow[t]{5}{*}{0.13331} & $0.2507(20)$ & $0.1840(10)$ & $0.2027(12)$ & $0.2190(22)$ & $0.1982(13)$ \\
\hline 2.1 & & $0.2340(28)$ & $0.1588(12)$ & $0.1798(12)$ & $0.1979(28)$ & $0.1736(15)$ \\
\hline 3.0 & & $0.2067(35)$ & $0.1258(15)$ & $0.1482(13)$ & $0.1674(34)$ & $0.1402(18)$ \\
\hline 5.0 & & $0.1671(56)$ & $0.0827(21)$ & $0.1056(17)$ & $0.1273(48)$ & $0.0944(25)$ \\
\hline 10.0 & & $0.112(11)$ & $0.0395(39)$ & $0.0581(23)$ & $0.0856(86)$ & $0.0427(44)$ \\
\hline 1.3 & \multirow[t]{5}{*}{0.13384} & $0.2515(24)$ & $0.1837(11)$ & $0.2033(15)$ & $0.2208(29)$ & $0.1976(16)$ \\
\hline 2.1 & & $0.2350(33)$ & $0.1585(15)$ & $0.1804(15)$ & $0.2000(36)$ & $0.1732(19)$ \\
\hline 3.0 & & $0.2079(43)$ & $0.1253(18)$ & $0.1488(17)$ & $0.1700(43)$ & $0.1397(23)$ \\
\hline 5.0 & & $0.1680(68)$ & $0.0823(26)$ & $0.1060(22)$ & $0.1303(62)$ & $0.0936(32)$ \\
\hline 10.0 & & $0.111(13)$ & $0.0399(46)$ & $0.0584(29)$ & $0.090(11)$ & $0.0407(55)$ \\
\hline 1.3 & \multirow[t]{5}{*}{0.13432} & $0.2526(29)$ & $0.1835(15)$ & $0.2031(20)$ & $0.2240(43)$ & $0.1970(23)$ \\
\hline 2.1 & & $0.2363(42)$ & $0.1581(19)$ & $0.1806(23)$ & $0.2040(53)$ & $0.1729(28)$ \\
\hline 3.0 & & $0.2094(55)$ & $0.1249(23)$ & $0.1488(25)$ & $0.1744(63)$ & $0.1395(34)$ \\
\hline 5.0 & & $0.1694(88)$ & $0.0820(33)$ & $0.1057(32)$ & $0.1346(91)$ & $0.0931(46)$ \\
\hline 10.0 & & $0.110(17)$ & $0.0404(59)$ & $0.0589(43)$ & $0.095(15)$ & $0.0389(77)$ \\
\hline 1.3 & \multirow[t]{5}{*}{$K_{s}$} & $0.2512(22)$ & $0.1839(11)$ & $0.2030(14)$ & $0.2203(27)$ & $0.1978(15)$ \\
\hline 2.1 & & $0.2346(31)$ & $0.1586(14)$ & $0.1801(14)$ & $0.1994(33)$ & $0.1734(18)$ \\
\hline 3.0 & & $0.2075(40)$ & $0.1255(17)$ & $0.1484(15)$ & $0.1692(40)$ & $0.1399(21)$ \\
\hline 5.0 & & $0.1677(64)$ & $0.0825(24)$ & $0.1058(21)$ & $0.1293(57)$ & $0.0939(29)$ \\
\hline 10.0 & & $0.112(13)$ & $0.0398(44)$ & $0.0583(27)$ & $0.088(10)$ & $0.0415(50)$ \\
\hline 1.3 & \multirow[t]{5}{*}{$K_{c}$} & $0.2544(40)$ & $0.1830(20)$ & $0.2037(29)$ & $0.2277(60)$ & $0.1959(33)$ \\
\hline 2.1 & & $0.2384(56)$ & $0.1575(25)$ & $0.1816(33)$ & $0.2087(75)$ & $0.1722(40)$ \\
\hline 3.0 & & $0.2117(75)$ & $0.1242(31)$ & $0.1497(36)$ & $0.1797(91)$ & $0.1388(48)$ \\
\hline 5.0 & & $0.171(12)$ & $0.0813(44)$ & $0.1061(46)$ & $0.141(13)$ & $0.0919(66)$ \\
\hline 10.0 & & $0.108(23)$ & $0.0412(78)$ & $0.0594(60)$ & $0.102(22)$ & $0.035(11)$ \\
\hline
\end{tabular}
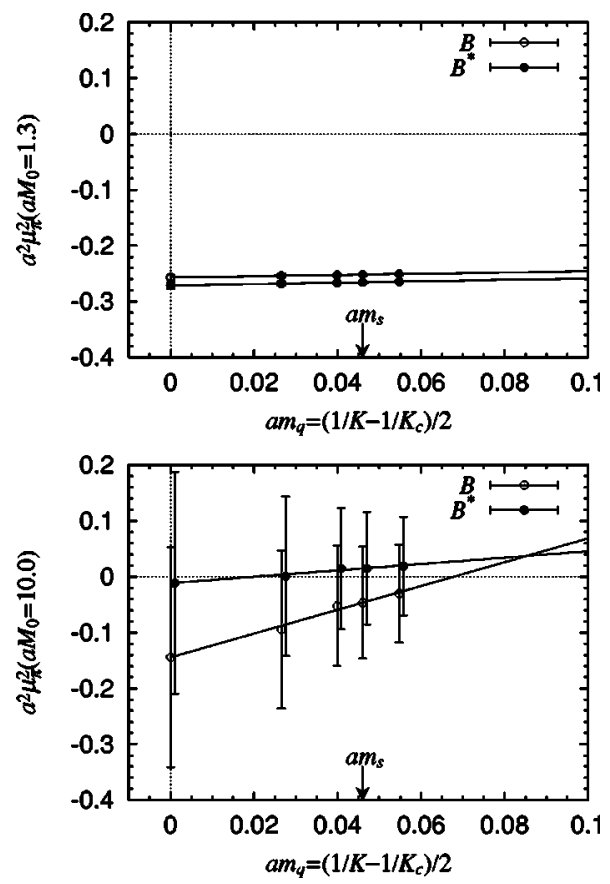

FIG. 7. Matrix element $\mu_{\pi}^{2}$ for the $B$ and $B^{*}$ mesons as a function of light quark mass at $a M_{0}=1.3$ (top panel) and 10.0 (bottom panel).
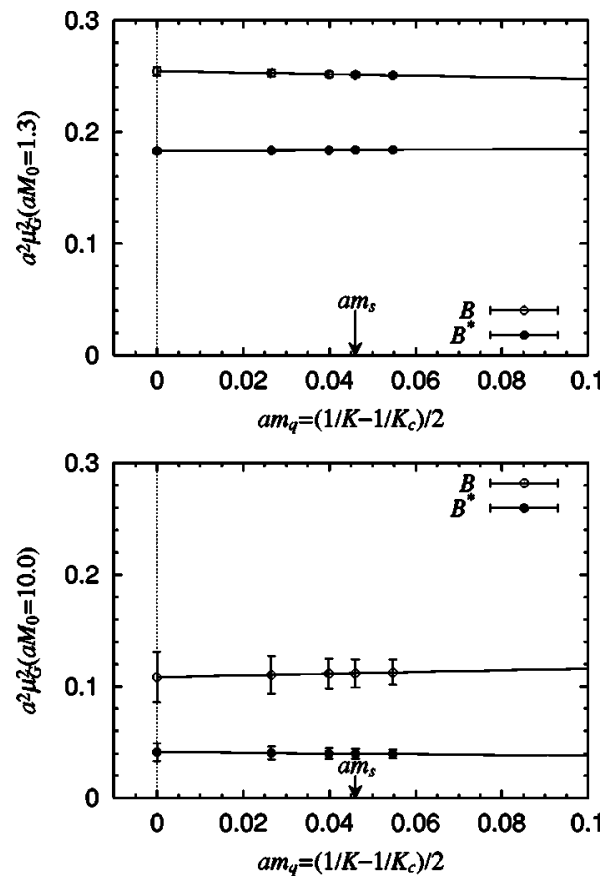

FIG. 8. Matrix element $\mu_{G}^{2}$ for the $B$ and $B^{*}$ mesons as a function of light quark mass at $a M_{0}=1.3$ (top panel) and 10.0 (bottom panel). 

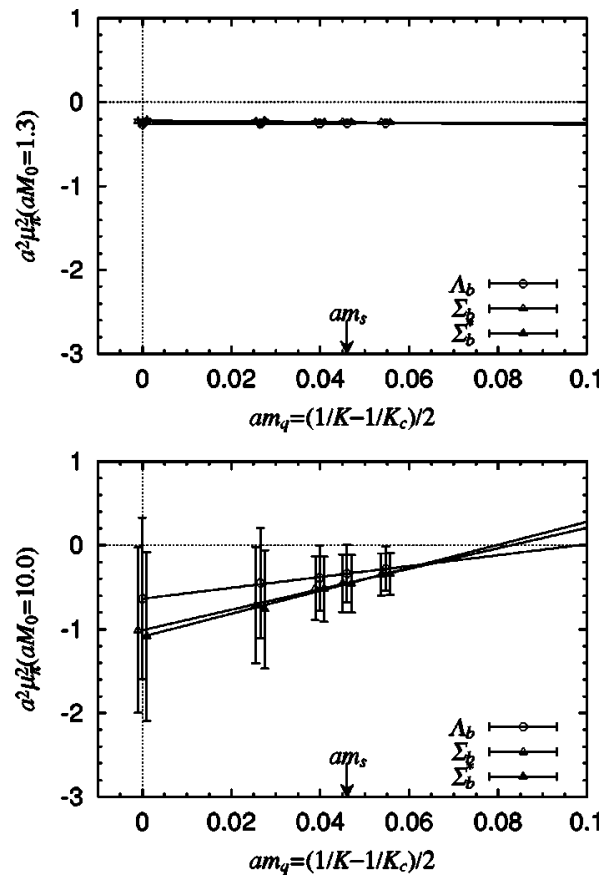

FIG. 9. Matrix element $\mu_{\pi}^{2}$ for the $\Lambda_{b}, \Sigma_{b}$ and $\Sigma_{b}^{*}$ baryons as a function of light quark mass at $a M_{0}=1.3$ (top panel) and 10.0 (bottom panel).

$$
O_{G}=\bar{Q}(\vec{\sigma} \cdot \vec{B}) Q .
$$

The interpolating operator $J$ is one of the operators listed in the previous subsection. The asymptotic behavior of the ratio yields the corresponding matrix element. We fix the position of the operator at $t^{\prime}=9$ and move the sink $t$.
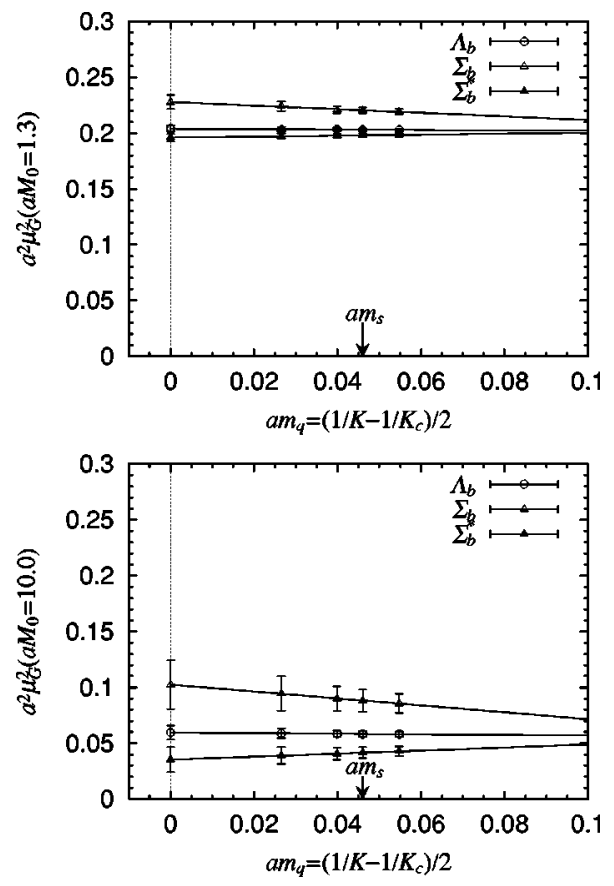

FIG. 10. Matrix element $\mu_{G}^{2}$ for the $\Lambda_{b}, \Sigma_{b}$ and $\Sigma_{b}^{*}$ baryons as a function of light quark mass at $a M_{0}=1.3$ (top panel) and 10.0 (bottom panel).

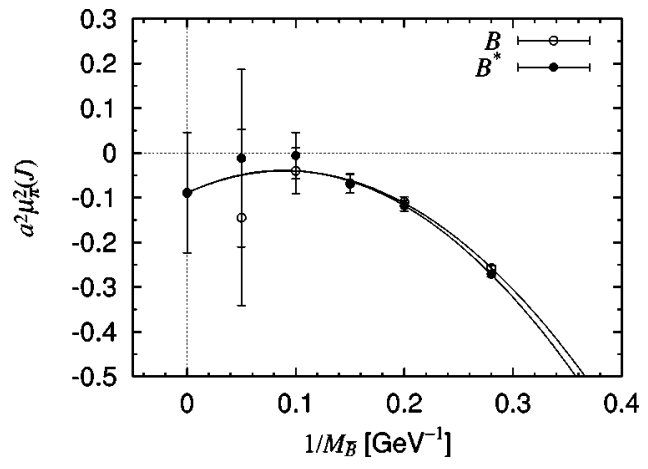

FIG. 11. Matrix element $\mu_{\pi}^{2}$ for the $B$ and $B^{*}$ mesons as a function of $1 / M_{\bar{B}}$. The value in the static limit is obtained from a fit with a quadratic function in $1 / M_{\bar{B}}$ with the constraint (5).

\section{E. Operator renormalization}

The matching of the operators $O_{\pi}$ and $O_{G}$ with their continuum counterpart has to be done in order to relate the matrix elements calculated on the lattice to the continuum quantities. The matching calculation can be carried out in perturbation theory. At present, however, the one-loop calculation is available only in the static limit $[28,29]$.

The perturbative expansion is poorly convergent for the kinetic operator $O_{\pi}$, since it mixes with lower dimensional operators $\bar{Q} D_{0} Q$ and $\bar{Q} Q$ as

$$
O_{\pi}^{c o n t}=Z_{\pi}\left(O_{\pi}^{l a t t}-\frac{c_{2}}{a^{2}}(\bar{Q} Q)^{l a t t}\right)
$$

in the static limit and a power divergence appears. Note that the operator $\bar{Q} D_{0} Q$ can be eliminated by using the equation of motion. Nonperturbative subtraction of the power divergent contribution $\left(c_{2} / a^{2}\right) \bar{Q} Q$ was attempted in [18], whereas in this paper we consider the physical quantities in which the power divergent term cancels. One of such quantities is the difference of the matrix elements between two hadron states such as $\mu_{\pi}^{2}\left(\Lambda_{b}\right)-\mu_{\pi}^{2}(B)$. The matrix element of the operator $\bar{Q} Q$ cancels at the leading order in $1 / m_{Q}$. The effect remains at finite values of $1 / m_{Q}$, and hence we take the

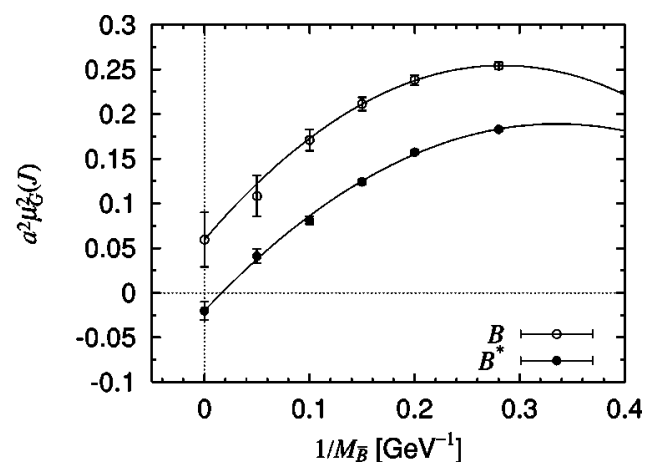

FIG. 12. Matrix element $\mu_{G}^{2}$ for the $B$ and $B^{*}$ mesons as a function of $1 / M_{\bar{B}}$. The values in the static limit are obtained from a fit with a quadratic function in $1 / M_{\bar{B}}$ with the constraint (6). 


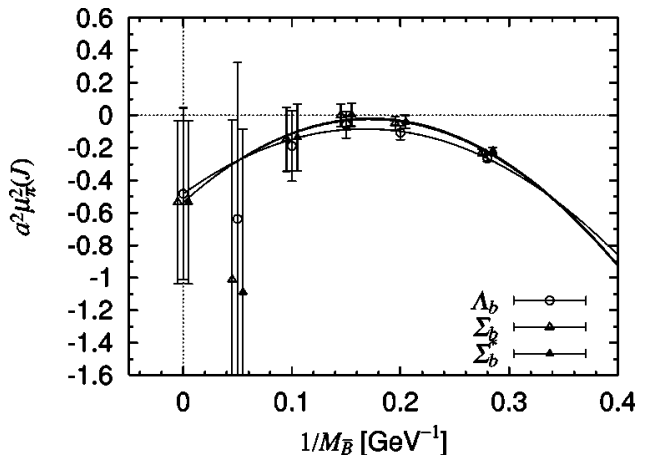

FIG. 13. Matrix element $\mu_{\pi}^{2}$ for the $\Lambda_{b}, \Sigma_{b}$ and $\Sigma_{b}^{*}$ baryons as a function of $1 / M_{\bar{B}}$.

infinite heavy quark mass limit after measuring the differences at several values of $m_{Q}$.

The multiplicative matching factor $Z_{\pi}$ is perturbatively calculated in the static limit in [28] as

$$
Z_{\pi}=1+0.0687 g^{2} \text {. }
$$

This quantity is also related to the mass renormalization factor $Z_{m}$ of the lattice NRQCD action. In the infinite mass limit one can expand the self-energy of heavy quark in terms of $1 / m_{Q}$, and then the kinetic term of heavy quark becomes an insertion of the operator $O_{\pi}$ for the amplitude in the static theory. For the heavy quark self-energy at $O\left(g^{2}\right)$

$$
\begin{aligned}
\Sigma(p)= & A a+B \frac{1}{2 m_{Q} a} \sum_{j}\left(2 \sin \frac{p_{i} a}{2}\right)^{2}+C\left[e^{i p_{0} a}-1\right. \\
& \left.+\frac{1}{2 m_{Q} a} \sum_{j}\left(2 \sin \frac{p_{i} a}{2}\right)^{2}\right]+\cdots,
\end{aligned}
$$

the relation is $Z_{\pi}=1+(B+A) g^{2}$. In the infinite mass limit $B+A=0.2370+(-0.1684)=0.0686$ for the NRQCD action of [30] in agreement with Eq. (43). Note that the definition of the coefficients $A$ and $B$ differ from that used in Table II by a factor $4 \pi$. For our choice of the NRQCD action the oneloop calculation of $B$ is not available in the infinite mass limit, but by an extrapolation from finite mass data given in Table II we obtain $B \simeq(-0.2) / 4 \pi$ and thus $B+A \simeq(-0.20$ $+1.01) / 4 \pi=0.069$. The power divergent coefficient $c_{2} / a^{2}$ in Eq. (42) is also related to $A$ and $C$.

Since we apply the tadpole improvement using the plaquette expectation value and its effect is to multiply the link variable by $1 / u_{0}$, the corresponding one-loop contribution $\frac{1}{12} g^{2}$ has to be subtracted from the one-loop coefficient, and thus we obtain

$$
\widetilde{Z}_{\pi}=1-0.0146 g^{2},
$$

whose numerical value at $\beta=6.0$ is 0.975 if we use the boosted coupling $\widetilde{g}^{2}=g_{0}^{2} / u_{0}^{4}=1.70$.

The other operator $O_{G}$ does not mix with lower dimensional ones in the static limit. However, once the $1 / m_{Q}$ correction is introduced, the mixing with $O_{\pi}$ and the other lower dimensional operators appears since the NRQCD ac-

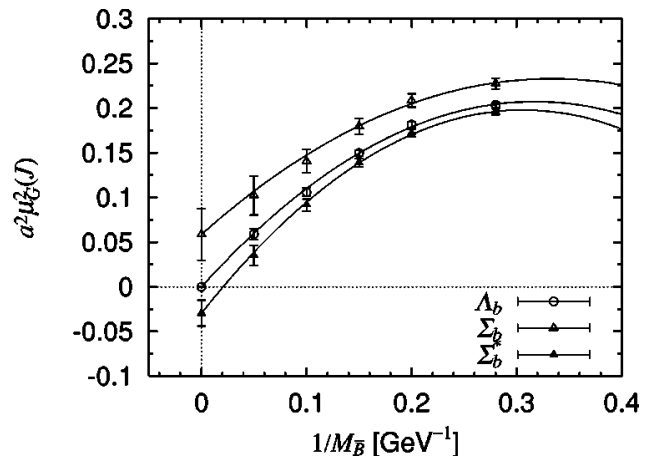

FIG. 14. Matrix element $\mu_{G}^{2}$ for the $\Lambda_{b}, \Sigma_{b}$ and $\Sigma_{b}^{*}$ baryons as a function of $1 / M_{\bar{B}}$.

tion contains the $\vec{\sigma} \cdot \vec{B}$ term. Hence, we again consider the difference among different hadron states to cancel the mixing contribution and take the infinite heavy quark mass limit.

The one-loop calculation of the multiplication renormalization is found in [29]:

$$
Z_{G}=1+g^{2}\left(-\frac{3}{16 \pi^{2}} \ln m_{Q}^{2} a^{2}+0.437\right),
$$

where $m_{Q}$ denotes the heavy quark mass arising from the continuum theory. The tadpole improvement amounts to multiply $1 / u_{0}^{4}$ and the one-loop coefficient is modified as

$$
\widetilde{Z}_{G}=1+g^{2}\left(-\frac{3}{16 \pi^{2}} \ln m_{Q}^{2} a^{2}+0.104\right),
$$

and its numerical value is 1.12 for the $b$ quark mass $m_{b}$ $=4.6 \mathrm{GeV}$. For both operators the tadpole improvement acts to greatly reduce the perturbative coefficients.

\section{RESULTS}

In this section, we present the results for hadron masses and matrix elements. The heavy quark mass dependence of the matrix elements from the direct calculation is studied carefully by two methods. We also make a comparison between the results from the direct calculation and from the indirect calculation. All errors of measured quantities are es-

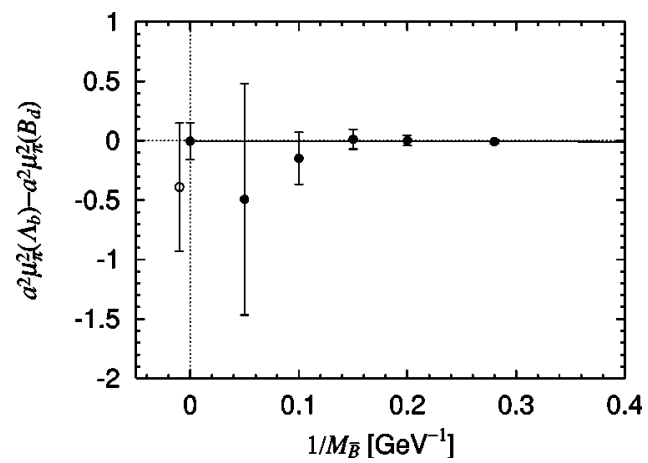

FIG. 15. Difference of the matrix element $\mu_{\pi}^{2}\left(\Lambda_{b}\right)-\mu_{\pi}^{2}\left(B_{d}\right)$ as a function of $1 / M_{\bar{B}}$. Open circle denotes the result from method 2 . 
TABLE VI. Matrix element $\mu_{\pi}^{2}$ for heavy-light mesons and heavy-light-light baryons in the static heavy quark limit.

\begin{tabular}{lcccc}
\hline \hline$a M_{0}$ & $K$ & $a^{2} \mu_{\pi}^{2}(B)=a^{2} \mu_{\pi}^{2}\left(B^{*}\right)$ & $a^{2} \mu_{\pi}^{2}\left(\Lambda_{b}\right)$ & $a^{2} \mu_{\pi}^{2}\left(\Sigma_{b}\right)=a^{2} \mu_{\pi}^{2}\left(\Sigma_{b}^{*}\right)$ \\
\hline static & 0.13331 & $-0.057(61)$ & $-0.25(17)$ & $-0.22(13)$ \\
static & $K_{s}$ & $-0.061(69)$ & $-0.29(21)$ & $-0.26(17)$ \\
static & $K_{c}$ & $-0.09(13)$ & $-0.48(53)$ & $-0.54(50)$ \\
\hline \hline
\end{tabular}

timated by the single elimination jackknife procedure.

\section{A. Hadron masses}

In Figs. 1 and 2 we show the typical effective mass plots for relevant mesons and baryons. The plateau is convincing for the $B$ and $B^{*}$ mesons (Fig. 1) in the time region starting around $t=8$, while it starts later in time for baryons (Fig. 2) and is dominated by statistical fluctuations after $t=20$. We therefore fit the data in the time interval $[10,20]$ for mesons and in $[12,20]$ for baryons. The results for the binding energy are summarized in Table III.

Because the light quark mass dependence of the binding energy is well described by a linear function as shown in Figs. 3 and 4, we can extrapolate (interpolate) the binding energy to the chiral limit (to the strange quark). The binding energy at the chiral limit and the strange quark is also presented in Table III.

\section{B. Matrix elements}

The ratio $R_{i}\left(J ; t, t^{\prime}\right)$ defined in Eq. (39) is shown as a function of $t$ in Fig. 5 for $B$ and $B^{*}$ mesons. It shows a statistically cleanest data with heaviest light $(K=0.13331)$ and lightest heavy $(a M=1.3)$ quarks. The plateau is very convincing and appears earlier in $R_{G}$ than in $R_{\pi}$, and then we fit the data with a constant in the time interval $[17,25]$ for $R_{\pi}$ or $[14,25]$ for $R_{G}$. For other mass parameters the data are noisier, but we can identify the plateau in the same time interval. Similar plots for baryons $\left(\Lambda_{b}, \Sigma_{b}\right.$, and $\left.\Sigma_{b}^{*}\right)$ are shown in Fig. 6. Since the statistical error dominates earlier in time we truncate the fit range at $t=23$. The results for the matrix elements $\mu_{\pi}^{2}$ and $\mu_{G}^{2}$ are summarized in Tables IV and $\mathrm{V}$, respectively.

From Figs. 7-10 we see that the light quark mass dependence of the matrix elements is mild though the statistical error grows as light quark mass decreases. We therefore take a simple linear fit in the light quark mass to obtain the results in the physical light quark mass.

On the other hand, the heavy quark mass dependence of the matrix elements is significant as shown in Figs. 11-14. In particular, the matrix elements $\mu_{G}^{2}(B)$ and $\mu_{G}^{2}\left(B^{*}\right)$ in Fig. 12 are both positive at finite heavy quark masses, and hence do not respect the symmetry relation $\frac{1}{3} \mu_{G}^{2}(B)=-\mu_{G}^{2}\left(B^{*}\right)$ given in Eq. (6). This is due to the effects of operator mixing of $\bar{Q} \vec{\sigma} \cdot \vec{B} Q$ with spin singlet operators as mentioned in the previous section. The similar violation of the relation (12) is found in Fig. 14 for the matrix elements of $\Sigma_{b}^{(*)}$ baryons $\mu_{G}^{2}\left(\Sigma_{b}\right)$ and $\mu_{G}^{2}\left(\Sigma_{b}^{*}\right)$.

In order to extract the prediction in the static limit, where the symmetry relations have to be satisfied, we perform a fit of data in terms of a quadratic function in $1 / M_{\bar{B}}$ with a constraint known in the static limit. For mesons the constraint is Eq. (5) or Eq. (6), while for baryons we may impose Eq. (11) or Eq. (12). The fitting curves describe the data well while satisfying the constraints as shown in Figs. 11-14. The bare matrix elements extrapolated to the static limit are listed in Tables VI and VII. Since the chromomagnetic operator $\bar{Q} \vec{\sigma}$ - $\vec{B} Q$ does not receive the additive renormalization in the static limit, we may extract the physical result from these numbers. We obtain

$$
\begin{array}{r}
\lambda_{2}(B)\left(\equiv \frac{1}{3} \mu_{G}^{2}(B)=-\mu_{G}^{2}\left(B^{*}\right)\right)=0.076(39) \mathrm{GeV}^{2}, \\
\mu_{G}^{2}\left(\Sigma_{b}\right)=-2 \mu_{G}^{2}\left(\Sigma_{b}^{*}\right)=0.23(11) \mathrm{GeV}^{2}
\end{array}
$$

after multiplying the renormalization factor $\widetilde{Z}_{G}=1.12$ defined in Eq. (47).

For the other matrix element $\mu_{\pi}^{2}$, the difference of the matrix elements between different heavy hadrons has to be considered in order to avoid the additive renormalization due to the mixing with lower dimensional operators. It also helps to reduce the statistical error as it correlates among different hadrons. The results are

$$
\mu_{\pi}^{2}\left(\Lambda_{b}\right)-\mu_{\pi}^{2}(B)=-1.3(1.8) \mathrm{GeV}^{2}
$$

TABLE VII. Matrix element $\mu_{G}^{2}$ for heavy-light mesons and heavy-light-light baryons in the static heavy quark limit.

\begin{tabular}{lcccccc}
\hline \hline$a M_{0}$ & $K$ & $a^{2} \mu_{G}^{2}(B)$ & $a^{2} \mu_{G}^{2}\left(B^{*}\right)$ & $a^{2} \mu_{G}^{2}\left(\Lambda_{b}\right)$ & $a^{2} \mu_{G}^{2}\left(\Sigma_{b}\right)$ & $a^{2} \mu_{G}^{2}\left(\Sigma_{b}^{*}\right)$ \\
\hline static & 0.13331 & $0.065(15)$ & $-0.022(05)$ & 0 & $0.039(11)$ & $-0.020(06)$ \\
static & $K_{s}$ & $0.064(17)$ & $-0.021(06)$ & 0 & $0.043(13)$ & $-0.021(07)$ \\
static & $K_{c}$ & $0.060(30)$ & $-0.020(10)$ & 0 & $0.059(29)$ & $-0.029(15)$ \\
\hline \hline
\end{tabular}




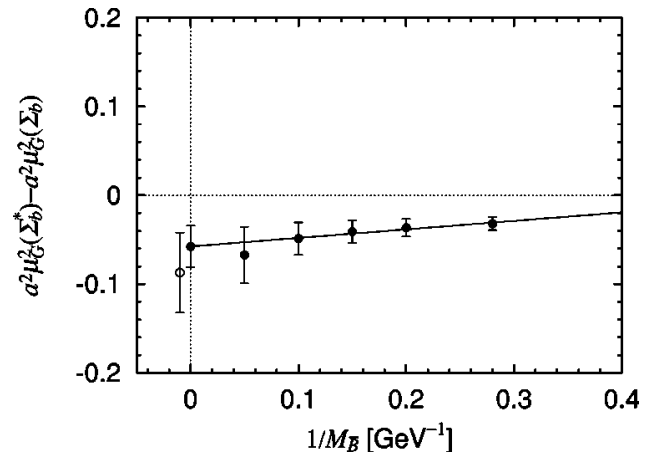

FIG. 16. Difference of the matrix element $\mu_{G}^{2}\left(\Sigma_{b}^{*}\right)-\mu_{G}^{2}\left(\Sigma_{b}\right)$ as a function of $1 / M_{\bar{B}}$. Open circle denotes the result from method 2 .

$$
\mu_{\pi}^{2}\left(\Sigma_{b}\right)-\mu_{\pi}^{2}\left(\Lambda_{b}\right)=-0.2(2.5) \mathrm{GeV}^{2}
$$

which include the multiplicative renormalization factor $\widetilde{Z}_{\pi}$ $=0.975$ as calculated in Eq. (45). The SU(3) breaking $\mu_{\pi}^{2}\left(B_{s}\right)-\mu_{\pi}^{2}\left(B_{d}\right)$ has also a phenomenological importance, as it appears in the evaluation of the lifetime ratio $\tau\left(B_{s}\right) / \tau\left(B_{d}\right)$. Our result is

$$
\mu_{\pi}^{2}\left(B_{s}\right)-\mu_{\pi}^{2}\left(B_{d}\right)=0.09(26) \mathrm{GeV}^{2}
$$

Another way to extract these physical quantities is to take the differences before extrapolating the data to the static limit. As an example, we plot the difference of the matrix element $\mu_{\pi}^{2}$ between $\Lambda_{b}$ baryon and $B$ meson in Fig. 15 . Since each matrix element $\mu_{\pi}^{2}\left(\Lambda_{b}\right)$ or $\mu_{\pi}^{2}(B)$ has a quite similar heavy quark mass dependence as seen in Figs. 11 and 13, the heavy quark mass dependence almost cancels in the difference (Fig. 15). We fit the data with a linear function in $1 / M_{\bar{B}}$ and obtain

$$
\mu_{\pi}^{2}\left(\Lambda_{b}\right)-\mu_{\pi}^{2}(B)=-0.01(52) \mathrm{GeV}^{2},
$$

in the static limit. This result is consistent with the previous analysis (50) within one standard deviation. Since the heavy quark mass dependence is numerically better controlled in this method, we quote Eq. (53) as our final result, while taking the other to estimate systematic uncertainty arising from the heavy quark extrapolation. The results for other differences of $\mu_{\pi}^{2}$ are

$$
\begin{aligned}
& \mu_{\pi}^{2}\left(\Sigma_{b}\right)-\mu_{\pi}^{2}\left(\Lambda_{b}\right)=0.28(68) \mathrm{GeV}^{2}, \\
& \mu_{\pi}^{2}\left(B_{s}\right)-\mu_{\pi}^{2}\left(B_{d}\right)=0.066(80) \mathrm{GeV}^{2} .
\end{aligned}
$$

The same strategy-differentiate then extrapolate-works even for $\mu_{G}^{2}$, since the additive renormalization at finite heavy quark masses mostly cancel in the differences like $\mu_{G}^{2}\left(B^{*}\right)-\mu_{G}^{2}(B)$ or $\mu_{G}^{2}\left(\Sigma_{b}^{*}\right)-\mu_{G}^{2}\left(\Sigma_{b}\right)$. Figure 16 shows the difference $\mu_{G}^{2}\left(\Sigma_{b}^{*}\right)-\mu_{G}^{2}\left(\Sigma_{b}\right)$ as a function of $1 / M_{\bar{B}}$. We find that the heavy quark mass dependence is much milder than the individual matrix elements as shown in Fig. 14. This cancellation of the $1 / M_{\bar{B}}$ dependence is easily understood from Fig. 12 or 14, because the mass dependence is similar for all heavy hadrons. The results are

$$
\lambda_{2}(B)=-\frac{1}{4}\left(\mu_{G}^{2}\left(B^{*}\right)-\mu_{G}^{2}(B)\right)=0.094(19) \mathrm{GeV}^{2},
$$

$$
\mu_{G}^{2}\left(\Sigma_{b}\right)=-\frac{2}{3}\left(\mu_{G}^{2}\left(\Sigma_{b}^{*}\right)-\mu_{G}^{2}\left(\Sigma_{b}\right)\right)=0.147(60) \mathrm{GeV}^{2},
$$

which are consistent with the results obtained by taking the difference after the extrapolation, Eqs. (48) and (49), respectively.

All these results are summarized in Table VIII, where "method 1" means our preferred method (differentiate-thenextrapolate) while "method 2" denotes the other (extrapolate-then-differentiate).

\section{Heavy quark expansion parameters from mass differences}

The parameters $\bar{\Lambda}, \mu_{\pi}^{2}$ and $\mu_{G}^{2}$ can also be indirectly obtained from hadron masses using the mass formula (4). We use the hadron masses measured on the lattice to obtain the HQE parameters.

TABLE VIII. Results for the HQE parameters.

\begin{tabular}{lccc}
\hline \hline & $\begin{array}{c}\text { Direct. calc. } \\
(\text { method 1,2) }\end{array}$ & Mass difference & Other works \\
\hline $\bar{\Lambda}\left(\Lambda_{b}\right)-\bar{\Lambda}(B)[\mathrm{MeV}]$ & & $428(68)$ & $415(156)[19]$ \\
$\bar{\Lambda}\left(\Sigma_{b}\right)-\bar{\Lambda}\left(\Lambda_{b}\right)[\mathrm{MeV}]$ & $96(96)$ & $176(152)[19]$ \\
$\bar{\Lambda}\left(B_{s}\right)-\bar{\Lambda}\left(B_{d}\right)\left[\mathrm{MeV}^{2}\right.$ & & $90(7)$ & $81(31)[19]$ \\
$\mu_{\pi}^{2}\left(\Lambda_{b}\right)-\mu_{\pi}^{2}(B)\left[\mathrm{GeV}^{2}\right]$ & $-0.01(52),-1.3(1.8)$ & $-0.38(47)$ & $0[19]$ \\
$\mu_{\pi}^{2}\left(\Sigma_{b}\right)-\mu_{\pi}^{2}\left(\Lambda_{b}\right)\left[\mathrm{GeV}^{2}\right]$ & $0.28(68),-0.2(2.5)$ & $0.29(66)$ & $0.0 .09(4)[18], 0.10(28)[19]$ \\
$\mu_{\pi}^{2}\left(B_{s}\right)-\mu_{\pi}^{2}\left(B_{d}\right)\left[\mathrm{GeV}^{2}\right]$ & $0.066(80), 0.09(26)$ & $0.056(42)$ & $0.09(19)$ \\
$\lambda_{2}\left(B_{d}\right)\left[\mathrm{GeV}^{2}\right]$ & $0.094(19), 0.076(39)$ & $0.051(16)$ & $0.070(15)[18], 0.069(19)[19]$ \\
$\lambda_{2}\left(B_{s}\right)\left[\mathrm{GeV}^{2}\right]$ & $0.090(10), 0.082(22)$ & $0.053(8)$ & $0.078(12)[19]$ \\
$\mu_{G}^{2}\left(\Sigma_{b}\right)\left[\mathrm{GeV}^{2}\right]$ & $0.147(60), 0.23(11)$ & $0.09(7)$ & $0.06(2)$ \\
\hline \hline
\end{tabular}




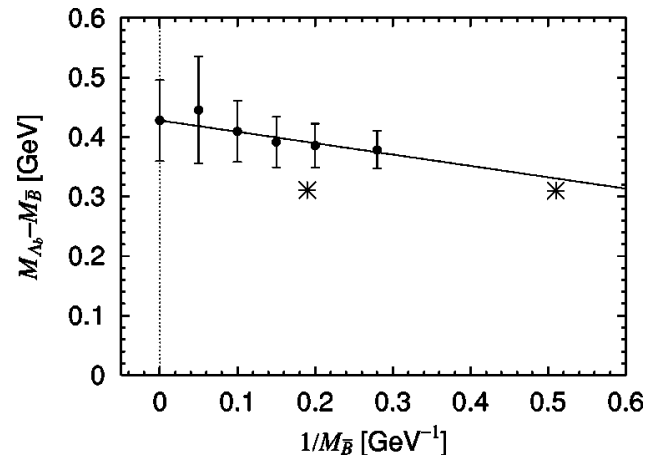

FIG. 17. $M_{\Lambda_{b}}-M_{\bar{B}}$ as a function of $1 / M_{\bar{B}}$. The light quark mass is extrapolated to the chiral limit.

We plot the mass difference $M_{\Lambda_{b}}-M_{\bar{B}}$ as a function of the spin-averaged meson mass inverse $1 / M_{\bar{B}}$ in Fig. 17. The mass formula (4) is given as an expansion in $1 / m_{Q}$, but here we analyze the data with $1 / M_{\bar{B}}$. The difference is of order $1 / m_{Q}^{2}$ which we neglect in this analysis. Fitting the data with a linear function of $1 / M_{\bar{B}}$ we obtain

$$
\bar{\Lambda}\left(\Lambda_{b}\right)-\bar{\Lambda}(B)=428(68) \mathrm{MeV},
$$

from the intercept. This result is in good agreement with a previous lattice calculation by Ali Khan et al., $\bar{\Lambda}\left(\Lambda_{b}\right)$ $-\bar{\Lambda}(B)=415$ (156) $\mathrm{MeV}$ [19]. Our result is slightly larger than the experimental value, which is about $310 \mathrm{MeV}$ for bottom and charmed hadrons as plotted in Fig. 17 by bursts. To draw a definite conclusion, however, we have to take account of several systematic errors. The finite volume effect is probably the most important one, because the physical extent of our lattice $\sim 2 \mathrm{fm}$ may not be large enough for baryons.

The slope of the mass difference $M_{\Lambda_{b}}-M_{\bar{B}}$ yields

$$
\mu_{\pi}^{2}\left(\Lambda_{b}\right)-\mu_{\pi}^{2}(B)=-0.38(47) \mathrm{GeV}^{2},
$$

which is compatible with the direct measurement of the matrix elements (53) and also with the phenomenological estimate $-0.01(3) \mathrm{GeV}^{2}[8]$ obtained from a combination $\left(M_{\Lambda_{b}}-M_{\bar{B}}\right)-\left(M_{\Lambda_{c}}-M_{\bar{D}}\right)$.

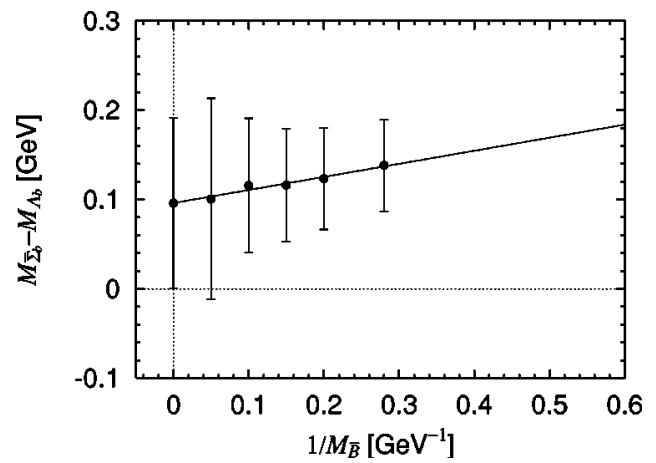

FIG. 18. $M_{\bar{\Sigma}_{b}}-M_{\Lambda_{b}}$ as a function of $1 / M_{\bar{B}}$. The light quark mass is extrapolated to the chiral limit.

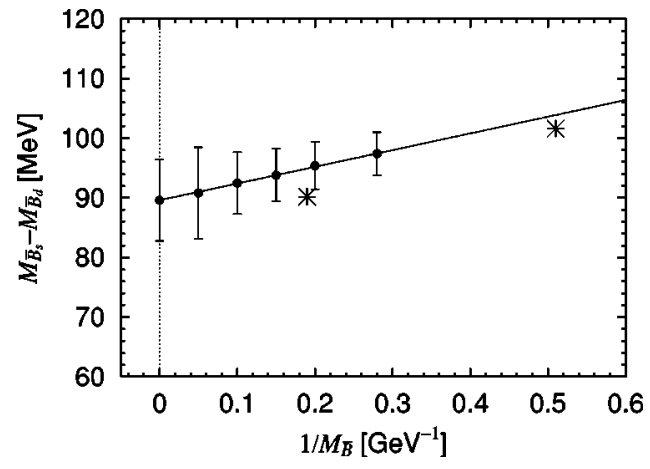

FIG. 19. $M_{\bar{B}_{s}}-M_{\bar{B}_{d}}$ as a function of $1 / M_{\bar{B}}$.

Similar analysis can be performed for $M_{\bar{\Sigma}_{b}}-M_{\Lambda_{b}}$, which is plotted in Fig. 18. We obtain

$$
\begin{aligned}
\bar{\Lambda}\left(\Sigma_{b}\right)-\bar{\Lambda}\left(\Lambda_{b}\right) & =96(96) \mathrm{MeV}, \\
\mu_{\pi}^{2}\left(\Sigma_{b}\right)-\mu_{\pi}^{2}\left(\Lambda_{b}\right) & =0.29(66) \mathrm{GeV}^{2},
\end{aligned}
$$

which are also consistent with the previous work $\bar{\Lambda}\left(\Sigma_{b}\right)$ $-\bar{\Lambda}\left(\Lambda_{b}\right)=176(152) \mathrm{MeV}$ and $\mu_{\pi}^{2}\left(\Sigma_{b}\right)-\mu_{\pi}^{2}\left(\Lambda_{b}\right) \sim 0$ [19].

The strange-nonstrange mass difference $M_{\bar{B}_{s}}-M_{\bar{B}_{d}}$ is plotted in Fig. 19. It is interesting to see that the data agree well with the experimental value for $B_{(s)}$ and $D_{(s)}$ mesons including the slope in $1 / M_{\bar{B}}$. A linear fit gives

$$
\begin{gathered}
\bar{\Lambda}\left(B_{s}\right)-\bar{\Lambda}\left(B_{d}\right)=90(7) \mathrm{MeV}, \\
\mu_{\pi}^{2}\left(B_{s}\right)-\mu_{\pi}^{2}\left(B_{d}\right)=0.056(42) \mathrm{GeV}^{2},
\end{gathered}
$$

which may be compared with $\bar{\Lambda}\left(B_{s}\right)-\bar{\Lambda}\left(B_{d}\right)$ $=81(31) \mathrm{MeV}$ and $\mu_{\pi}^{2}\left(B_{s}\right)-\mu_{\pi}^{2}\left(B_{d}\right)=0.10(28) \mathrm{GeV}^{2}$ obtained in [19].

The hyperfine splitting in the mesons $M_{B_{d}^{*}}-M_{B_{d}}$ and $M_{B_{s}^{*}}-M_{B_{s}}$ and in the baryons $M_{\Sigma_{b}}^{*}-M_{\Sigma_{b}}$ is plotted in Figs. 20,21 and 22 , respectively, as a function of $1 / M_{\bar{B}}$. The numerical values at each quark masses are given in Table IX, where the statistical error in the hyperfine splittings is greatly reduced because it is highly correlated within the spin multiplets.

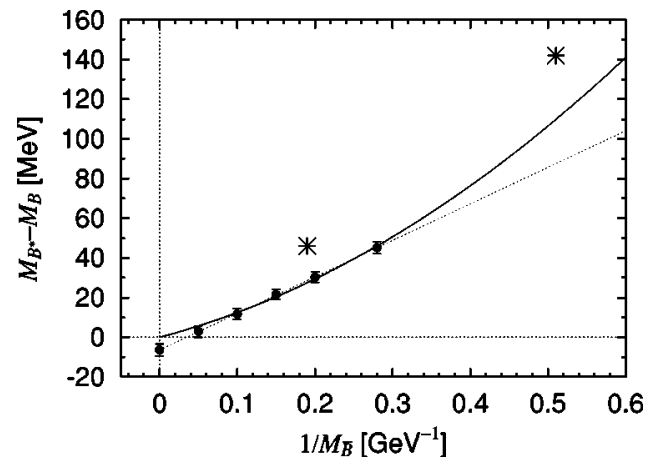

FIG. 20. Hyperfine splitting $M_{B^{*}}-M_{B}$ as a function of $1 / M_{\bar{B}}$. The light quark mass is extrapolated to the chiral limit. 


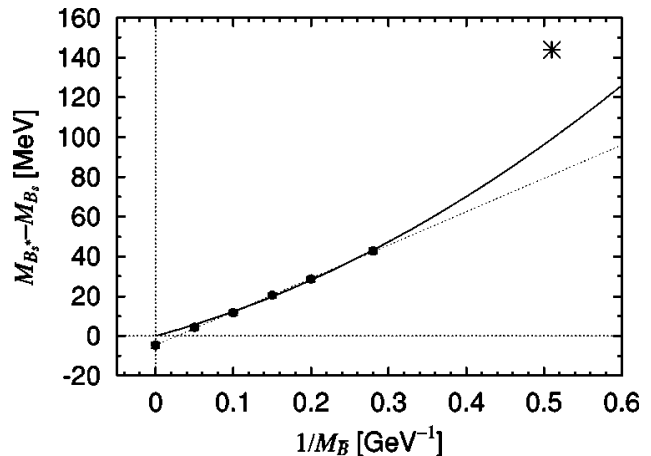

FIG. 21. Hyperfine splitting $M_{B_{s}^{*}}-M_{B_{s}}$ as a function of $1 / M_{\bar{B}}$. The light quark mass is interpolated into the strange quark mass.

For the $B-B^{*}$ splitting (Fig. 20) we observe a linear behavior which is consistent with the expectation that the hyperfine splitting is proportional to $1 / m_{Q}$. The intercept at $1 / M_{\bar{B}}$ is, however, slightly negative. Since the hyperfine splitting is exactly zero in the static limit, we attempt a constrained fit with a linear and quadratic terms in $1 / M_{\bar{B}}$, which is also shown in Fig. 20. It indicates that the quadratic term is not negligible and amounts about $5 \%$ at the $B$ meson mass. From the coefficient of the linear term we obtain

$$
\lambda_{2}\left(B_{d}\right)=0.051(16) \mathrm{GeV}^{2} .
$$

The similar analysis for $B_{s}$ gives

$$
\lambda_{2}\left(B_{s}\right)=0.053(8) \mathrm{GeV}^{2} .
$$

The data and fit curves are shown in Fig. 21. For the baryon hyperfine splitting $M_{\Sigma_{b}^{*}}-M_{\Sigma_{b}}$ shown in Fig. 22, the statistical error is so large that the intercept of the linear fit is statistically consistent with zero. The slope yields

$$
\mu_{G}^{2}\left(\Sigma_{b}^{*}\right)-\mu_{G}^{2}\left(\Sigma_{b}\right)=-0.13(11) \mathrm{GeV}^{2} .
$$

The experimental values of $M_{B^{*}}-M_{B}, M_{D^{*}}-M_{D}$, $M_{B_{s}^{*}}-M_{B_{s}}$ and $M_{\Sigma_{c}^{*}}-M_{\Sigma_{c}}$ are also shown in Figs. 20, 21 and 22. ( $M_{\Sigma_{b}^{*}}-M_{\Sigma_{b}}$ has not yet been measured.) The lattice data are significantly lower than these experimental results as in many other quenched lattice calculations. This is partly

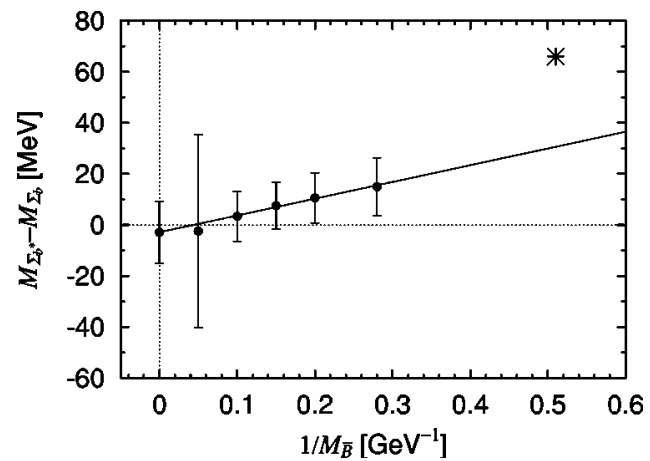

FIG. 22. Hyperfine splitting $M_{\Sigma_{b}^{*}}-M_{\Sigma_{b}}$ as a function of $1 / M_{\bar{B}}$. The light quark mass is extrapolated to the chiral limit.

due to the fact that the spin-chromo-magnetic interaction term in the lattice NRQCD action (20) is matched to the continuum full theory only at the tree level, although the mean field improvement is applied. The one-loop matching coefficient is known in the static limit as given in Eq. (47). Since the hyperfine splitting is proportional to the chromomagnetic interaction, we expect an increase of order $10 \%$ for $\lambda_{2}$, which is not enough to explain the discrepancy with the phenomenological values. Another important uncertainty is in the quenching approximation, whose effect is not yet entirely uncovered.

The numerical results given in this subsection are also summarized in Table VIII together with the results from other groups and the experimental values.

\section{Consistency among matrix elements and mass differences}

Results presented so far indicate that the HQE parameters are determined consistently with the direct measurement of the matrix elements and with the indirect measurement through the mass differences. However, more stringent test is possible using the data at fixed light quark mass, whose statistical error is smaller than in the chiral limit. Although the numerical values are unphysical, there is nothing wrong in the consistency check. For this purpose we use the data at $K=0.13331$, which corresponds to the heaviest light quark mass.

From Eqs. (7) and (8) the hyperfine splitting $M_{B^{*}}-M_{B}$ is given by $4 \lambda_{2} / 2 m_{b}$, or up to higher order $1 / m_{b}$ corrections,

TABLE IX. Mass difference between heavy hadrons.

\begin{tabular}{lccccc}
\hline \hline$a M_{0}$ & $K$ & $a\left(M_{B^{*}}-M_{B}\right)$ & $a\left(M_{\Sigma_{b}^{*}}-M_{\Sigma_{b}}\right)$ & $a\left(M_{\Lambda_{b}}-M_{\bar{B}}\right)$ & $a\left(M_{\bar{\Sigma}_{b}}-M_{\Lambda_{b}}\right)$ \\
\hline 1.3 & $K_{s}$ & $0.0231(08)$ & $0.0062(21)$ & $0.2851(63)$ & $0.055(09)$ \\
2.1 & & $0.0155(07)$ & $0.0043(18)$ & $0.2852(72)$ & $0.052(11)$ \\
3.0 & & $0.0111(07)$ & $0.0031(17)$ & $0.2855(81)$ & $0.051(12)$ \\
5.0 & & $0.0063(07)$ & $0.0014(18)$ & $0.288(11)$ & $0.048(15)$ \\
10.0 & & $0.0024(08)$ & $-0.0003(27)$ & $0.297(18)$ & $0.046(24)$ \\
1.3 & $K_{c}$ & $0.0244(16)$ & $0.0081(61)$ & $0.205(17)$ & $0.075(28)$ \\
2.1 & & $0.0164(14)$ & $0.0058(53)$ & $0.209(20)$ & $0.067(31)$ \\
3.0 & & $0.0116(14)$ & $0.0041(50)$ & $0.212(23)$ & $0.063(34)$ \\
5.0 & $0.0063(15)$ & $0.0018(53)$ & $0.221(28)$ & $0.063(41)$ \\
10.0 & & $0.0016(16)$ & $-0.001(20)$ & $0.241(49)$ & $0.055(61)$ \\
\hline \hline
\end{tabular}




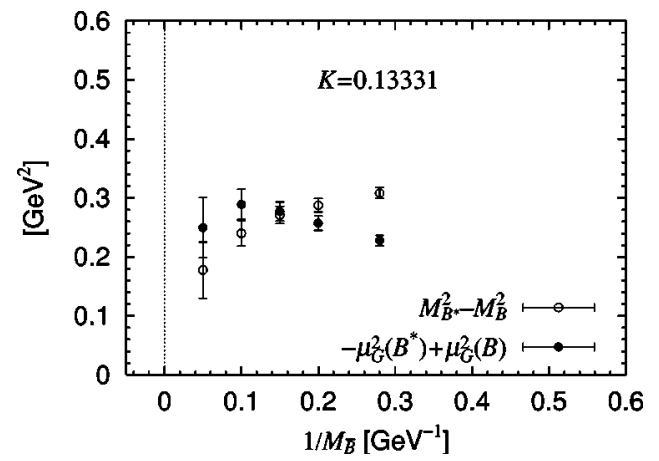

FIG. 23. Hyperfine splitting of the heavy-light ground state mesons as a function of $1 / M_{\bar{B}}$.

$$
M_{B *}^{2}-M_{B}^{2}=-\mu_{G}^{2}\left(B^{*}\right)+\mu_{G}^{2}(B) .
$$

In Fig. 23, we plot the results for $-\mu_{G}^{2}\left(B^{*}\right)+\mu_{G}^{2}(B)$ as a function of $1 / M_{\bar{B}}$ together with the lattice measurement of $M_{B *}^{2}-M_{B}^{2}$. We observe that the relation (67) is satisfied well in the heavy quark mass region $1 / M_{\bar{B}}<0.2 \mathrm{GeV}^{-1}$. Toward lighter heavy quark mass the data deviate from the relation (67), which is an indication of higher order effect. Similar analysis can be done for the hyperfine splitting of heavylight-light baryon, i.e., the $\Sigma_{b}^{*}-\Sigma_{b}$ splitting. Figure 24 shows the mass difference and the matrix element $-\Delta \mu_{G}^{2}$. Both are in good agreement within the large statistical error in the hadron mass measurement.

The heavy-light meson-baryon mass difference $M_{\Lambda_{b}}$ $-M_{\bar{B}}$ is given as

$$
M_{\Lambda_{b}}-M_{\bar{B}}=\bar{\Lambda}\left(\Lambda_{b}\right)-\bar{\Lambda}(B)+\frac{1}{2 m_{b}}\left[\mu_{\pi}^{2}\left(\Lambda_{b}\right)-\mu_{\pi}^{2}(B)\right] .
$$

In Fig. 25 we plot $M_{\Lambda_{b}}-M_{\bar{B}}$ as a function of $1 / M_{\bar{B}}$. The slope obtained from the fit of the mass difference yields an indirect estimate of $\mu_{\pi}^{2}\left(\Lambda_{b}\right)-\mu_{\pi}^{2}(B)$ as -0.03 $\pm 0.15 \mathrm{GeV}^{2}$. Our results for the direct measurement of $\mu_{\pi}^{2}\left(\Lambda_{b}\right)-\mu_{\pi}^{2}(B)$ are plotted in Fig. 26, where the indirect measurement is shown by a band. Both measurements are completely consistent with each other.

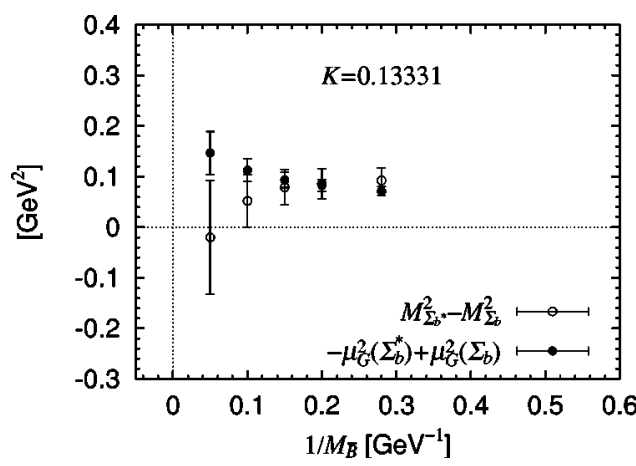

FIG. 24. Hyperfine splitting of the heavy-light-light baryons as a function of $1 / M_{\bar{B}}$.

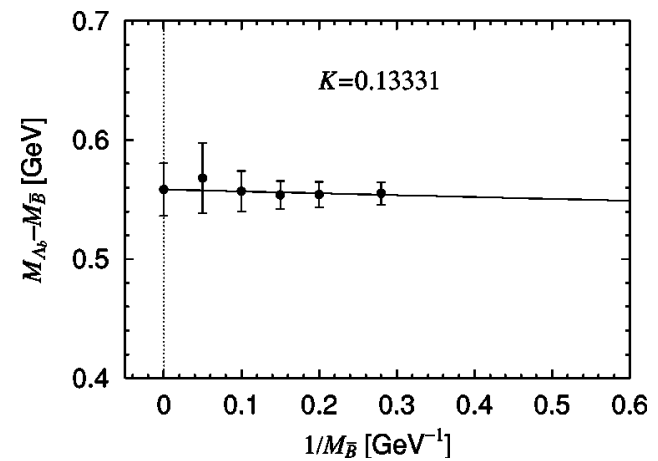

FIG. 25. $M_{\Lambda_{b}}-M_{\bar{B}}$ as a function of $1 / M_{\bar{B}}$.

\section{LIFETIME RATIO: A PHENOMENOLOGICAL APPLICATION}

In the ratio of lifetimes of different $b$ hadrons $H_{b}^{(1)}$ and $H_{b}^{(2)}$ the hadronic matrix elements $\mu_{\pi}^{2}$ and $\mu_{G}^{2}$ appear as

$$
\begin{aligned}
\frac{\tau\left(H_{b}^{(1)}\right)}{\tau\left(H_{b}^{(2)}\right)}= & 1+\frac{\mu_{\pi}^{2}\left(H_{b}^{(1)}\right)-\mu_{\pi}^{2}\left(H_{b}^{(2)}\right)}{2 m_{b}^{2}} \\
& +c_{G} \frac{\mu_{G}^{2}\left(H_{b}^{(1)}\right)-\mu_{G}^{2}\left(H_{b}^{(2)}\right)}{m_{b}^{2}}+O\left(\frac{1}{m_{b}^{3}}\right),
\end{aligned}
$$

with a perturbative coefficient $c_{G} \simeq 1.2$ [8]. Our calculation of the differences of the matrix elements $\mu_{\pi}^{2}\left(H_{b}^{(1)}\right)$ $-\mu_{\pi}^{2}\left(H_{b}^{(2)}\right)$ and $\mu_{G}^{2}\left(H_{b}^{(1)}\right)-\mu_{G}^{2}\left(H_{b}^{(2)}\right)$ may be directly used to evaluate the lifetime ratios at the order $1 / m_{b}^{2}$.

Using our results $\mu_{\pi}^{2}\left(\Lambda_{b}\right)-\mu_{\pi}^{2}\left(B_{d}\right)=-0.01(52) \mathrm{GeV}^{2}$ and $\mu_{G}^{2}\left(\Lambda_{b}\right)-\mu_{G}^{2}\left(B_{d}\right)\left[\equiv-3 \lambda_{2}\left(B_{d}\right)\right]=-0.282(59) \mathrm{GeV}^{2}$, which are from the direct calculation (method 1), the lifetime ratio of $\Lambda_{b}$ and $B_{d}$ is evaluated as

$$
\frac{\tau\left(\Lambda_{b}\right)}{\tau\left(B_{d}\right)}=0.984 \pm 0.012 \pm 0.003+O\left(\frac{1}{m_{b}^{3}}\right)
$$

with $m_{b}=4.6 \mathrm{GeV}$, where the first and second error comes from the statistical error of $\mu_{\pi}^{2}\left(\Lambda_{b}\right)-\mu_{\pi}^{2}\left(B_{d}\right)$ and $\mu_{G}^{2}\left(\Lambda_{b}\right)$ $-\mu_{G}^{2}\left(B_{d}\right)$, respectively. As discussed in the previous works [8] it may not explain the experimental value $0.76(5)$ unless

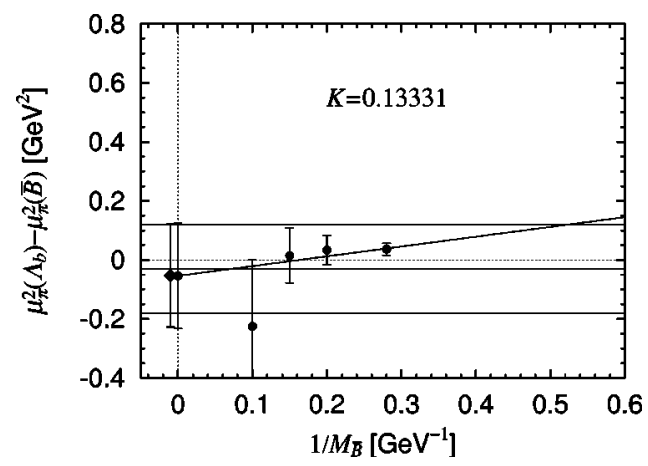

FIG. 26. $-\mu_{\pi}^{2}\left(\Lambda_{b}\right)+\mu_{\pi}^{2}(\bar{B})$ measured from the matrix elements is compared with the indirect measurement from the slope of mass difference $M_{\Lambda_{b}}-M_{\bar{B}}$, which gives $-0.03 \pm 0.15 \mathrm{GeV}^{2}$. 
the higher order effect in the $1 / m_{b}$ expansion has a substantially large effect. Our calculation does not imply such a large correction to the matrix element $\mu_{\pi}^{2}$ as shown in Fig. 26. At the order $1 / m_{b}^{3}$ the spectator effect arises, for which the hadronic matrix elements of higher dimensional operators are necessary [8]. A lattice calculation [31] of those matrix elements suggests that the spectator effects are indeed significant but do not appear to be sufficiently large to account for the full discrepancy.

The lifetime ratio of $B_{s}$ and $B_{d}$ is obtained as

$$
\frac{\tau\left(B_{s}\right)}{\tau\left(B_{d}\right)}=1.001 \pm 0.002 \pm 0.002+O\left(\frac{1}{m_{b}^{3}}\right)
$$

using our lattice results $\mu_{\pi}^{2}\left(B_{s}\right)-\mu_{\pi}^{2}\left(B_{d}\right)$ $=0.066(80) \mathrm{GeV}^{2} \quad$ and $\quad \mu_{G}^{2}\left(B_{s}\right)-\mu_{G}^{2}\left(B_{d}\right)$ $=-0.012(32) \mathrm{GeV}^{2}$. This result may be compared with the experimental value $\tau\left(B_{s}\right) / \tau\left(B_{d}\right)=0.949 \pm 0.038$ [32].

\section{CONCLUSIONS}

In this article we present a lattice QCD calculation of the heavy quark expansion parameters $\bar{\Lambda}, \mu_{\pi}^{2}$ and $\mu_{G}^{2}$ for the heavy-light mesons and heavy-light-light baryons. The lattice NRQCD action is used for heavy quark and the results in the static limit are obtained by an extrapolation.

For $\mu_{\pi}^{2}$ and $\mu_{G}^{2}$, we performed a direct calculation of the matrix elements through the three-point functions. While the light quark mass dependence of the matrix elements is small, the heavy quark mass dependence is significant due to the effect of the additive renormalization. The large heavy quark mass dependence mostly cancels by considering the difference of the matrix elements between different heavy hadron states, in which the additive renormalization cancels. We also estimate the differences of the HQE parameters by studying the mass differences between several heavy hadrons.

We find that the lattice measurements of the matrix elements $\mu_{\pi}^{2}$ and $\mu_{G}^{2}$ are consistent with the mass relations predicted by the heavy quark expansion. Our numerical results for the differences of $\mu_{\pi}^{2}$ in the heavy quark mass limit are compatible with the previous determinations from the meson mass spectrum. The deficit of the hyperfine splitting - the well-known problem of the quenched lattice calculation-is also reproduced in the direct calculation of the matrix element $\mu_{G}^{2}$.

A direct phenomenological application of our results is the evaluation of the lifetime ratios at the order $1 / \mathrm{m}_{b}^{2}$. Previously such analysis implicitly assumed that the heavy quark expansion truncated at $1 / m_{b}^{2}$ is valid down to the charm quark mass, as the parameter $\mu_{\pi}^{2}$ was determined using the combined mass difference including charmed mesons and baryons. Through the direct lattice calculation we have confirmed that such analysis is justified. The problem of the small lifetime ratio $\tau\left(\Lambda_{b}\right) / \tau\left(B_{d}\right)$ still remains.

\section{ACKNOWLEDGMENTS}

This work was supported by the Supercomputer Project No. 66 (FY2001) and No. 79 (FY2002) of High Energy Accelerator Research Organization (KEK), and also in part by the Grants-in-Aid of the Ministry of Education (Nos. $10640246,11640294,12014202,12640253,12640279$, $12740133,13135204,13640259,13640260,14046202$, 14740173). N.Y. is supported by the JSPS.
[1] M. Neubert, Adv. Ser. Dir. High Energy Phys. 15, 239 (1998).

[2] I.I. Bigi, M.A. Shifman, and N. Uraltsev, Annu. Rev. Nucl. Part. Sci. 47, 591 (1997).

[3] J. Chay, H. Georgi, and B. Grinstein, Phys. Lett. B 247, 399 (1990)

[4] I.I. Bigi, M.A. Shifman, N.G. Uraltsev, and A.I. Vainshtein, Phys. Rev. Lett. 71, 496 (1993).

[5] A.V. Manohar and M.B. Wise, Phys. Rev. D 49, 1310 (1994).

[6] B. Blok, L. Koyrakh, M.A. Shifman, and A.I. Vainshtein, Phys. Rev. D 49, 3356 (1994); 50, 3572(E) (1994).

[7] G. Martinelli and C.T. Sachrajda, Phys. Lett. B 354, 423 (1995).

[8] M. Neubert and C.T. Sachrajda, Nucl. Phys. B483, 339 (1997).

[9] S. Hashimoto, Phys. Rev. D 50, 4639 (1994).

[10] R.D. Dikeman, M.A. Shifman, and N.G. Uraltsev, Int. J. Mod. Phys. A 11, 571 (1996).

[11] A. Kapustin and Z. Ligeti, Phys. Lett. B 355, 318 (1995).

[12] A.F. Falk, M.E. Luke, and M.J. Savage, Phys. Rev. D 53, 2491 (1996).

[13] A.F. Falk, M.E. Luke, and M.J. Savage, Phys. Rev. D 53, 6316 (1996).
[14] M. Gremm, A. Kapustin, Z. Ligeti, and M.B. Wise, Phys. Rev. Lett. 77, 20 (1996).

[15] P. Ball and V.M. Braun, Phys. Rev. D 49, 2472 (1994).

[16] M. Neubert, Phys. Lett. B 389, 727 (1996).

[17] M. Crisafulli, V. Gimenez, G. Martinelli, and C.T. Sachrajda, Nucl. Phys. B457, 594 (1995).

[18] V. Gimenez, G. Martinelli, and C.T. Sachrajda, Nucl. Phys. B486, 227 (1997).

[19] A. Ali Khan et al., Phys. Rev. D 62, 054505 (2000).

[20] A.S. Kronfeld and J.N. Simone, Phys. Lett. B 490, 228 (2000); 495, 441(E) (2000).

[21] B.A. Thacker and G.P. Lepage, Phys. Rev. D 43, 196 (1991).

[22] G.P. Lepage, L. Magnea, C. Nakhleh, U. Magnea, and K. Hornbostel, Phys. Rev. D 46, 4052 (1992).

[23] JLQCD Collaboration, K.I. Ishikawa et al., Phys. Rev. D 61, 074501 (2000).

[24] JLQCD Collaboration, S. Aoki et al., hep-lat/0208038.

[25] G.P. Lepage and P.B. Mackenzie, Phys. Rev. D 48, 2250 (1993).

[26] B. Sheikholeslami and R. Wohlert, Nucl. Phys. B259, 572 (1985). 
[27] M. Luscher, S. Sint, R. Sommer, P. Weisz, and U. Wolff, Nucl. Phys. B491, 323 (1997).

[28] L. Maiani, G. Martinelli, and C.T. Sachrajda, Nucl. Phys. B368, 281 (1992).

[29] J.M. Flynn and B.R. Hill, Phys. Lett. B 264, 173 (1991).
[30] C.T.H. Davies and B.A. Thacker, Phys. Rev. D 45, 915 (1992).

[31] UKQCD Collaboration, M. Di Pierro, C.T. Sachrajda, and C. Michael, Phys. Lett. B 468, 143 (1999).

[32] B Lifetime Working group, http://lepbosc.web.cern.ch/ LEPBOSC/lifetimes/lepblife.html 